\title{
1 The discovery of gene mutations making SARS-CoV-2 \\ 2 well adapted for humans: host-genome similarity 3 analysis of 2594 genomes from China, the USA and \\ 4 Europe
}

6 Weitao $\operatorname{Sun}^{1,2 * \&}$

$7 \quad{ }^{1}$ School of Aerospace Engineering, Tsinghua University, Beijing, 100084, China

$8{ }^{2}$ Zhou Pei-Yuan Center for Applied Mathematics, Tsinghua University, Beijing, 100084, China

9

$10 *$ Corresponding author

11 Email: sunw@tsinghua.edu.cn

13 Short title

14 Discovery of SARS-CoV-2 gene mutations by host-genome similarity analysis 


\section{Abstract}

20 Severe acute respiratory syndrome coronavirus 2 (SARS-CoV-2), a positive-sense single-stranded virus

21 approximately $30 \mathrm{~kb}$ in length, causes the ongoing novel coronavirus disease-2019 (COVID-19). Studies

22 confirmed significant genome differences between SARS-CoV-2 and SARS-CoV, suggesting that the

23 distinctions in pathogenicity might be related to genomic diversity. However, the relationship between

24 genomic differences and SARS-CoV-2 fitness has not been fully explained, especially for open reading frame

25 (ORF)-encoded accessory proteins. RNA viruses have a high mutation rate, but how SARS-CoV-2 mutations

26 accelerate adaptation is not clear. This study shows that the host-genome similarity (HGS) of SARS-CoV-2

27 is significantly higher than that of SARS-CoV, especially in the ORF6 and ORF8 genes encoding proteins

28 antagonizing innate immunity in vivo. A power law relationship was discovered between the HGS of ORF3b,

29 ORF6, and $\mathrm{N}$ and the expression of interferon (IFN)-sensitive response element (ISRE)-containing

30 promoters. This finding implies that high HGS of SARS-CoV-2 genome may further inhibit IFN I synthesis

31 and cause delayed host innate immunity. An ORF1ab mutation, 10818G $>\mathrm{T}$, which occurred in virus

32 populations with high HGS but rarely in low-HGS populations, was identified in 2594 genomes with

33 geolocations of China, the USA and Europe. The $10818 \mathrm{G}>\mathrm{T}$ caused the amino acid mutation M37F in the

34 transmembrane protein nsp6. The results suggest that the ORF6 and ORF8 genes and the mutation M37F

35 may play important roles in causing COVID-19. The findings demonstrate that HGS analysis is a promising

36 way to identify important genes and mutations in adaptive strains, which may help in searching potential

37 targets for pharmaceutical agents.

\section{Introduction}

39 In December 2019, a novel coronavirus SARS-CoV-2 was reported as the cause of COVID-19. SARS-CoV-2

40 has a positive-sense single-stranded RNA with a length of approximately $30 \mathrm{~kb}[1]$. Studies have shown that

41 considerable genetic diversity exists between SARS-CoV-2 and SARS-CoV[1, 2]. Compared with SARS- 
$42 \mathrm{CoV}, \mathrm{SARS}-\mathrm{CoV}-2$ appears to be more contagious and more adapted to humans[3]. The distinctions in

43 pathogenicity and virulence might be related to genomic diversity.

44 RNA viruses are susceptible to genetic recombination, and viral populations may evolve improved

45 adaptability in the process of infecting hosts. By comparing the genome similarity of the virus to the host,

46 the adaptability of the virus to the host can be inferred. Although the genomes of viruses and hosts are quite

47 different in general, nucleotide sequence similarities do exist. Such similarities may have three biological

48 significances. (1) These similar fragments come from a common ancestor and remain stable over long-term

49 evolution due to their biological significance. (2) Similar genomic fragments are coincidentally preserved in

50 both viruses and hosts over time because of the biological benefits of the gene products. (3) When the virus

51 interacts with the hosts, mutants are created by virus-host gene exchanges, causing genome similarities.

52 A growing number of studies on virus-host gene similarity have been reported. Simian virus 40 (SV40), the

53 first animal virus to undergo complete full-sequence DNA analysis, can infect monkeys and humans and

54 cause tumors[4]. Rosenberg et al.[5] found that some mutant SV40 viruses contained nucleic acid sequences

55 from their host monkeys. This finding suggests that viruses can recombine with host genes to complete their

56 own physiological processes, which makes up for a lack of function or increases virulence. Genes similar to

57 specific fragments of the human genome in molluscum contagiosum virus (MCV) have been reported[6].

$58 \mathrm{MCV}$ is a human poxvirus and lacks the genes associated with virus-host interactions in other poxvirus

59 species (variola virus). However, genes in MCV with high similarity to specific fragments of the human

60 genome are also hard to find in other poxviruses. These host-like genes may provide MCV-specific strategies

61 for coexistence with the host[6]. In other words, it is very likely that viruses use host-specific genes to perform

62 activities related to virus-host interactions, such as evasion of the host innate immune system. When human

63 peripheral blood DNA was used as a template for polymerase chain reaction (PCR), 5 of 6 samples could be

64 amplified by Epstein-Barr virus (EBV)- or hepatitis C virus (HCV)-specific primers[7]. Therefore, it is

65 speculated that some genes of the two viruses may also exist in the human genome or that the viruses may 
66 have homology with human genes. This hypothesis implies that not only can the virus have the host's genes

67 but also the host itself may have genes from the virus.

68 Selection pressure exerted by the host immune system plays an important role in shaping virus

69 mutations. Homology between virus and host proteins indicates the presence of host gene capture.

70 Evolution of viral genes may involve intergenome gene transfer and intragenome gene

71 duplication[8]. By acquiring immune modulation genes from cells, viruses have evolved proteins

72 that can regulate or inhibit the host's immune system[9, 10]. A recent study showed that human

73 genome evolution was shaped by viral infections[11]. In mammals, nearly $30 \%$ of the adaptive

74 amino acid changes in the human proteome are caused by viruses, suggesting that viruses are one

75 of the major driving factors for the evolution of mammalian and human proteomes[12]. These

76 findings support the possibility that SARS-CoV-2 may exchange genetic information with host

77 cells. It can be inferred that most of the traits and mechanisms retained in "coevolution" between

78 viruses and their hosts, including genetic and mutational mechanisms, benefit at least one or both.

79 At the molecular level of evolution, the exchange of genetic information is necessary for virus-host

80 mutual adaptation, leading to the similarity of nucleotide sequences.

81 It is interesting to study the relationship between gene similarities and viral

82 transmission/pathological ability. The single-stranded RNA of coronavirus generally encodes three

83 categories of proteins: (1) the replication proteins open reading frame (ORF)1a and ORF1ab; (2)

84 the structural proteins S (spike), E (envelope), M (membrane) and N (nucleocapsid); and (3)

85 accessory proteins with unknown homologues. The structural protein genes are organized as '-S-

86 E-M-N-' in the SARS-CoV-2 genome, and accessory protein genes are distributed between S and

87 E, $\mathrm{M}$ and $\mathrm{N}$. 
88 The accessory protein genes play a key role in inhibiting the innate immune response in vivo and

89 are more susceptible than the other genes to species-specific mutations under the pressure of

90 evolutionary selection. Once inside the cell, the virus immediately confronts other critical proteins

91 known as host-restriction factors (HRFs)[13]. HRFs are proteins that recognize and block viral

92 replication. Virus-host interactions control species specificity and viral infection ability. Under

93 pressure from the host immune system, viruses must be able to overcome a range of constraints

94 associated with the host species and often show evolutionary mutation selections. It is hypothesized

95 that accessory ORFs may retain beneficial mutations to increase host-genome similarity (HGS).

96 Identifying emerging genetic mutations in virus populations with high HGS may aid the

97 understanding of how SARS-CoV-2 evolved adaptation to humans. To the best of our knowledge,

98 studies on the genetic similarity between SARS-CoV-2 and the human genome have not been

99 reported.

100 This study investigated the HGS of SARS-CoV-2 genes and elucidated the links between HGS and

101 virus adaptation to humans. A power law relationship was discovered between the expression of

102 genes with interferon (IFN)-stimulated response elements (ISREs) and HGS. ORFs with higher

103 HGS suppressed the gene expression of ISRE-regulated genes to a greater extent. Applying HGS

104 analysis to 2594 SARS-CoV-2 genomes from China, the USA and Europe, it was found that the

105 ORF6 and ORF8 genes of SARS-CoV-2 had more significant HGS increments than SARS-CoV.

106 In addition, three different sets of surviving mutations were identified in SARS-CoV-2 genomes

107 for China, the USA and Europe. Interestingly, an ORF1ab mutation, 10818G $>\mathrm{T}$, which resulted in

108 the residue mutation M37F in the transmembrane protein nsp6, was observed in virus populations

109 of all three regions. This mutation did not occur in strain populations with low HGS but gradually

110 appeared in populations with high HGS. This finding provides strong evidence that SARS-CoV-2 
111 may accelerate adaptation in humans through increasing HGS of the ORF6 and ORF8 genes and

112 selecting the M37F mutation. However, the underlying mechanism by which these genes and

113 mutations make SARS-CoV-2 more adapted to humans remains unclear.

\section{Materials and Methods}

\section{Viral genome data}

116 By using BLAST ORFfinder[14], 31 ORFs were detected in the RNA genome sequence (29903 nt) of SARS-

117 CoV-2 (GenBank: MN908947.3). Only ATG was used as the ORF start codon, and nested ORFs were

118 ignored. Among all the ORFs in the SARS-CoV-2 sequence, we selected the longest 14 as targets, whose

119 lengths were no less than 75 nt. For genome comparison, ORFs in the SARS-CoV genome with a length of

$12029728 \mathrm{nt}$ (GenBank: AY394850.2) were also identified. There were 19 ORFs with lengths no less than $75 \mathrm{nt}$

121 in the SARS-CoV sequence.

122 The SARS-CoV-2 genomes were obtained from the GISAID database[15]. By May 20, 2020, the GISAID

123 database (https://www.gisaid.org/) had 416 SARS-CoV-2 genomes from China, 5184 genomes from the USA

124 and 10954 genomes from Europe. Complete and high-coverage genomes were used to ensure accurate HGS

125 calculations. The sequences containing nucleotide names other than A, G, C and T were removed from the

126 dataset. In total, 2594 SARS-CoV-2 genomes were used in the current study, including 200 from China, 1538

127 from the USA and 856 from Europe. The CDSs of the SARS-CoV-2 genome were identified by using

128 MATLAB (https://www.mathworks.com/help/bioinfo/ref/seqshoworfs.html). The accession IDs of the

129 genomes used in the article can be found in the Supplemental Information.

130 Human SARS-CoV genomes were collected from NCBI GenBank[16]. There were 25 CDSs of SARS-CoVs

131 (full-length sequences only, with all ORF sequences, no nucleotide names other than A, G, C and T) at the

132 time of article preparation. The accession IDs of these viral sequences can be found in the Supplemental

133 Information. 


\section{Host-genome similarity (HGS)}

135 The target CDSs were aligned with the human genome (Homo sapiens GRCh38.p12 chromosomes) by

136 Blastn[17] to obtain matching fragments. Blastn sequence alignment gives an original score of S. To facilitate

137 the comparison of Blast results among different subgenomic groups, the original score is standardized to S'

138 by Blastn:

$$
S^{\prime}=\frac{\lambda S-\ln K}{\ln 2}
$$

$$
E=m n 2^{-S^{\prime}}
$$

142 are matched and the score is not lower than $S^{\prime}$. Parameters $K$ and $\lambda$ describe the statistical significance of the

143 results[18]. Assuming that the fragment of length $a$ matches perfectly in the two random sequences, one has

144 the following formula:

$$
E=(m-a)(n-a) 4^{-a} \text {. }
$$

146 Since the viral genome is quite different from the human genome, matching fragments are usually very short.

147 When $a$ is particularly small compared to $m$ and $n, a=S^{\prime} / 2$ is obtained by combining Equation (3) and

148 Equation (4). Thus, HGS is defined as

$$
H=\frac{\sum a}{n}=\frac{\sum S^{\prime}}{2 n},
$$

150 where $n$ represents the length of the target sequence. The meaning of $H$ is the ratio of the number of 151 matched base pairs to the total length of the sequence when the matched sequences are converted into 152 sequences of the same length. 


\section{Data availability}

154 The SARS-CoV-2 genomes used in this study can be obtained at GISAID website (https://www.gisaid.org/).

155 The SARS-CoV genomes can be obtained at NCBI database (https://www.ncbi.nlm.nih.gov/). The accession 156 number and corresponding HGS of 2594 SARS-CoV-2 genomes and those of 25 SARS-CoV genomes are 157 in Supplemental Information. The code for HGS calculation is available in GitHub 158 (https://github.com/WeitaoNSun/HGS).

\section{Results}

\section{SARS-CoV-2 ORFs have higher HGS than those of SARS-CoV}

162 were used as references to establish the genome organization. SARS-CoV-2 has 14 5'-ORFs, while SARS-

163 CoV has $195^{\prime}$-ORFs. The length of each ORF is no less than $75 \mathrm{nt}$ (Table 1).

164 A quantitative definition of HGS was proposed to investigate the similarity between viral coding sequences

165 (CDSs) and the human genome (Homo sapiens GRCh38.p12 chromosomes). The CDS alignment scores were 166 determined by using NCBI Blastn[17], and HGS was calculated by the formulas described in the Methods 167 for each ORF in the coronavirus genome. The overall HGS of a full-length virus genome was obtained by 168 the weighted sum of ORF HGSs. The weighting factor was the ratio of ORF length to the full-genome length.

169 The ORF lengths of SARS-CoV and SARS-CoV-2 genomes are given in Table 1.

170 Table 1. Location, length and residue number of each ORF of SARS-CoV-2 and SARS-CoV genomes.

171 The ORF names defined in different papers are listed in the first three columns $(9,18,19)$.

\begin{tabular}{l|l|l|l|l|l|l|l|l|l|l}
\hline \multicolumn{2}{l|}{ ORF names } & \multicolumn{3}{l|}{ SARS-CoV } & \multicolumn{3}{l}{ SARS-CoV-2 } \\
\hline Narayanan & Marra & Rota & start & stop & length & residues & start & stop & length & residues \\
\hline ORF1a & ORF1a & la & 3361 & 13413 & 10053 & 3350 & 266 & 13483 & 13218 & 4405 \\
\hline N/R & N/R & N/R & 13685 & 13759 & 75 & 24 & 13685 & 13759 & 75 & 24 \\
\hline
\end{tabular}




\begin{tabular}{l|l|l|l|l|l|l|l|l|l|l}
\hline ORF1b & ORF1b & $1 \mathrm{~b}$ & 13398 & 21485 & 8088 & 2628 & 13768 & 21555 & 7788 & 2595 \\
\hline $\mathrm{S}$ & Sprotein & $\mathrm{S}$ & 21492 & 25259 & 3768 & 1282 & 21536 & 25384 & 3849 & 1282 \\
\hline $\mathrm{N} / \mathrm{R}$ & $\mathrm{N} / \mathrm{R}$ & $\mathrm{N} / \mathrm{R}$ & 25207 & 25329 & 123 & 40 & 25332 & 25448 & 117 & 38 \\
\hline ORF3a & ORF3 & $\mathrm{X} 1$ & 25268 & 26092 & 825 & 274 & 25393 & 26220 & 828 & 275 \\
\hline $\mathrm{E}$ & Eprotein & $\mathrm{E}$ & 26117 & 26347 & 231 & 76 & 26245 & 26472 & 228 & 75 \\
\hline $\mathrm{M}$ & Mprotein & $\mathrm{M}$ & 26398 & 27063 & 666 & 221 & 26523 & 27191 & 669 & 222 \\
\hline ORF6 & ORF7 & $\mathrm{X} 3$ & 27074 & 27265 & 192 & 63 & 27202 & 27387 & 186 & 61 \\
\hline ORF7a & ORF8 & $\mathrm{X} 4$ & 27273 & 27641 & 369 & 122 & 27394 & 27759 & 366 & 121 \\
\hline ORF7b & ORF9 & $\mathrm{N} / \mathrm{R}$ & 27638 & 27772 & 135 & 44 & 27756 & 27887 & 132 & 43 \\
\hline ORF8a & ORF10 & $\mathrm{N} / \mathrm{R}$ & 27779 & 27853 & 75 & 24 & 27894 & 28259 & 366 & 121 \\
\hline ORF8b & ORF11 & $\mathrm{X} 5$ & 27862 & 28116 & 255 & 84 & $\mathrm{~N} / \mathrm{R}$ & $\mathrm{N} / \mathrm{R}$ & $\mathrm{N} / \mathrm{R}$ & $\mathrm{N} / \mathrm{R}$ \\
\hline $\mathrm{N}$ & $\mathrm{Nprotein}$ & $\mathrm{N}$ & 28118 & 29386 & 1269 & 422 & 28274 & 29533 & 1260 & 419 \\
\hline $\mathrm{N} / \mathrm{R}$ & $\mathrm{N} / \mathrm{R}$ & $\mathrm{N} / \mathrm{R}$ & 29413 & 29490 & 78 & 25 & 29558 & 29674 & 117 & 38 \\
\hline
\end{tabular}

173 The HGS of ORFs was calculated for 2594 SARS-CoV-2 genomes with geolocation from China, the USA

174 and Europe. Phylogenetic trees representing the HGS relationship among virus strains are shown in Fig 1,

175 Fig 2 and Fig 3 for all three regions. The tree clusters were formed based on the distance between vectors

176 containing ORF HGS values. Most of the genomes had moderate HGS values. Genomes with similar HGS

177 values were usually in the same cluster and shared a common ancestor. The genomes with high HGS were

178 not all concentrated in the same cluster but may form several separate populations in the tree.

Fig 1. The HGS tree contains 200 SARS-CoV-2 genomes from China. Distance between leaves

is the unweighted pair distance between the 10-ORF-HGS vector of genomes. The color bar 
188 bar represents the overall HGS value of each genome (weighted sum of ORF HGS). Out of a

189 total of 1538 viral genomes, 140 have unique ORF HGS values. The histogram at the top left

190 shows the distribution of all genome HGS.

192 Fig 3. The HGS tree contains 856 SARS-CoV-2 genomes from Europe. Distance between

193 leaves is the unweighted pair distance between the 10-ORF-HGS vector of genomes. The color

194 bar represents the overall HGS value of each genome (weighted sum of ORF HGS). Out of a

195 total of 856 viral genomes, 98 have unique ORF HGS values. The histogram at the top left

196 shows the distribution of all genome HGS.

197 The full-length genome data were obtained from the Global Initiative on Sharing All Influenza Data

198 (GISAID) database[15]. The sequence requirements were full-length sequences only, sequences with definite

199 collection dates and locations, and no nucleotide names other than A, G, C and T. The number of genomes

200 that met such requirements was 200 for China, 1538 for the USA and 856 for Europe at the time of article

201 preparation. The HGS of human SARS-CoV genomes was also calculated. In NCBI GenBank[16], a total of

20225 SARS-CoV CDSs met the above sequence requirements.

203 Fig 4 shows that ORF 7b of SARS-CoV had the highest similarity with the human genome, followed by

204 ORF6, ORF7a, ORF3a and ORF 8. For SARS-CoV-2, ORF 7b, ORF 6 and ORF 8 were the top 3 genes with

205 the highest HGSs. The mean HGS values of ORF6 and ORF8 in SARS-CoV-2 increased significantly,

206 reaching $122 \%$ and $148 \%$ of those of SARS-CoV ORF6 and ORF8, respectively Fig 4 . The roles of such

207 HGS changes are not clear. However, by investigating the function of the SARS-CoV viral genes and

208 proteins, the mechanism of the rapid spread of the newly emerged COVID-19 may be inferred from the HGS

209 changes in SARS-CoV-2 genomes.

210 
211 Fig 4. The HGS values of SARS-CoV-2 and SARS-CoV genes. ORF6 and ORF8 of SARS-

212 CoV-2 have apparently higher mean HGS values than those of SARS-CoV, reaching 122\%

213 and $148 \%$ of that of SARS-CoV ORF6 and ORF8, respectively.

214 Studies have shown that ORF6 suppresses the induction of IFN and signaling pathways[19]. A membrane

215 protein with 63 amino acids, ORF 6 blocked the IFNAR-STAT signaling pathway by limiting the mobility

216 of the importin subunit KPNB1 and preventing the STAT1 complex from moving into the nucleus for ISRE

217 activation[20]. Laboratory studies confirmed that the expression of ORF 6 transformed a sublethal infection

218 into lethal encephalitis and enhanced the growth of the virus in cells[21]. In addition, ORF 6 circumvented

219 IFN production by inhibiting IRF-3 phosphorylation in the (TRAF3)-(TBK1+IKKe)-(IRF3)-(IFN $\beta$ )

220 signaling pathway (Fig 5), which is an essential signaling pathway triggered by the viral sensors RIG-

221 1/MDA5 and TLRs[22].

Fig 5. SARS-CoV induced immune response in host cells. Host cell detect virus invasion mainly by TLPs and RIG1/MDA5 and lead to type I IFN signaling pathway. The receptor IFNAR senses type I IFN and leads to the JAK1-STAT signaling pathway, which expresses antiviral proteins and bring neighboring cell into anti-virus state. The ORF6 suppresses type I IFN expression by inhibiting translocation of STAT1+STAT2+IRF9 complex into nucleus.

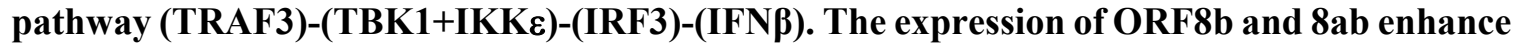

230 the IRF3 degradation, thus regulating immune functions of IRF3. 
233 CoV-infected cells[24]. Wong et al.[25] found that the proteins ORF8b and ORF8ab in SARS-CoV inhibited

234 the IFN response during viral infection. It was also reported that ORF8b formed insoluble intracellular

235 aggregates and triggered cell death[26]. Amazingly, studies showed that SARS-CoV-related CoVs in

236 horseshoe bats had 95\% genome identities to human and civet SARS-CoVs, but the ORF8 protein amino

237 acid similarities varied from $32 \%$ to $81 \%[27]$. These findings indicate that the ORF8 gene is more prone than

238 other $\mathrm{CoV}$ genes to mutations in virus-host interactions. Overexpression of ORF $8 \mathrm{~b}$ and ORF $8 \mathrm{ab}$ had a

239 significant effect on IRF3 dimerization rather than IRF3 phosphorylation[25]. The 8b region of SARS-CoV

240 protein ORF8 functions in ubiquitination binding, ubiquitination and glycosylation, which may interact with

241 IRF3[28]. The expression of ORF8b and 8ab enhanced IRF3 degradation, thus regulating the immune

242 functions of IRF3 (Fig 5). Interestingly, ORF8 is an IFN antagonist expressed in the later stage of SARS-

$243 \mathrm{CoV}$ infection. Studies showed that activation of IRF3 was blocked in the late stage of SARS-CoV infection,

244 which was consistent with the late expression of ORF8b. Therefore, the expression of ORF8 may help to

245 suppress the innate immune response that occurs in the later stages of infection and delay IFN $\beta$ signaling.

246 This may explain why the virus expresses a late-stage IFN antagonist, such as ORF8.

247 This work found that genes with high HGS were critical in suppressing innate immunity. Studies have shown

248 that the ORF3b, ORF6 and N proteins of SARS-CoV enhance suppression of IFN $\beta$ expression in host innate

249 immunity[29]. When IFN binds to the cell receptor IFNAR, the JAK/STAT signaling pathway is activated,

250 leading to activation of IFN-stimulated genes (ISGs) containing an ISRE in their promoter. Expression of

251 genes with an ISRE will trigger the production of hundreds of antiviral proteins inhibiting viral infections.

252 Therefore, a reduction in expression from ISRE-containing promoters is a direct indicator of the enhanced

253 ability to inhibit IFN synthesis.

254 ISRE-containing promoter expression after Sendai virus infection needs both IFN synthesis and signaling.

255 However, ISRE-containing promoter expression after IFN $\beta$ treatment requires only IFN signaling. In cells

256 treated with IFN $\beta$, it was found that $\mathrm{N}$ did not significantly inhibit the expression of the ISRE promoter[29].

257 The expression level was approximately $78 \%$ of the value for the empty control. However, ORF3b and ORF6 
258 still inhibited the expression of the ISRE promoter. We calculated the HGSs of ORF3b, ORF6 and N for

259 SARS-CoV. Amazingly, the results clearly demonstrated that the ISRE-containing promoter expression

260 decreased rapidly with increasing HGS (Fig 6), which provided evidence that there was a power law

261 dependence of IFN synthesis inhibition based on HGS. The ISRE-containing promoter expression data

262 followed the work of Kopecky-Bromberg et al.[29]. For 293T cells transfected with the SARS-CoV proteins

263 and infected by Sendai virus[29], IFN inhibition obeys the following power law equation:

$264 P=0.004 H^{-0.539}+5.421$, where $H$ is the HGS value of the viral genes ORF $3 b$, ORF6 and N, and $P$ is

265 the expression of genes with an ISRE as a percentage of the value for the empty control. The power law

266 equation for cells treated with IFN $\beta$ is $P=0.00001 H^{-11.007}+3.633$. The coefficient of determination $R^{2}$

267 reaches 1 for both data sets, indicating a perfect fit for the power law dependence on HGS.

Fig 6. Inhibition of a promoter containing an ISRE by SARS-CoV proteins with different genome HGS values. Cells were cotransfected with the SARS-CoV proteins and either 272 the promoter decays rapidly with the increasing HGS of ORF 3b, ORF 6 and N, conforming

\section{3 to a power law.}

274 The findings suggested that HGS, i.e., similarity between the virus and host genome, is a reliable indicator 275 of the suppression of innate immunity by viral proteins. Channappanavar et al. found that rapid SARS-CoV 276 replication and a relative delay in IFN I signaling resulted in immune dysregulation and severe disease in 277 infected mice[30]. Considering the significant HGS increments of ORF6 and ORF8 and their roles in 278 suppressing innate immunity, it could be speculated that SARS-CoV-2 would further suppress IFN I 279 synthesis and delay host innate immunity as HGS increases. This hypothesis may explain the delayed immune 280 response and uncontrolled inflammatory response that lead to the epidemiological manifestations of SARS- 
$281 \mathrm{CoV}-2$, such as long incubation periods, mild symptoms, rapid spread and low mortality. However, the

282 mechanism of how viral proteins cause further delay of immune signaling and how it leads to new

283 immunopathological features remain largely unknown.

284 The discovery of increased HGS of ORF 6 and ORF 8 provides strong evidence that SARS-CoV-2 evolved

285 to be more adapted to humans than SARS-CoV. These inferences offer a valuable picture of how SARS-

286 CoV-2 could have become different from SARS-CoV. In addition, genetic mutations making the virus

287 genome adapted to humans can also be identified through HGS analysis.

\section{The SARS-CoV-2 mutation 10818G $>$ T is adapted to humans}

Recent studies have shown that SARS-CoV-2 had a high mutation rate, and new mutations have emerged in ORF1ab, S, ORF3a and ORF8[31, 32]. However, the types of mutations that contribute to viral adaptations in humans are not clear. To understand how mutations aid survival of SARS-CoV-2 populations under

292 selective pressure, the accumulated nucleotide variants in consensus sequences were identified in 2594

293 genomes from China, the USA and Europe. The virus genome was identified by its HGS values of ten ORFs 294 (ORF1ab, S, ORF3a, E, M, ORF6, ORF7a, ORF7b, ORF8, and N). The percentages of virus strains with 295 unique ORF HGSs were 18\% (36 out of 200), 9\% (140 out of 1538) and 11\% (98 out of 856) for genomes 296 with geolocations of China, the USA and Europe, respectively. A total of 74 mutations, 162 mutations and 297145 mutations were identified in genomes for these three regions, respectively. Gene mutation profiles of 298 SARS-CoV-2 genomes with different HGSs are shown inFig 6, Fig 7 and Fig 8. SARS-CoV-2 in different 299 regions developed its own conserved mutations independently (Table 2). For example, the mutations in 300 genomes with a geolocation of China included the ORF1ab mutations 10818G $>$ T (TTG>TTT), 1132G $>$ A 301 (GTA $>$ ATA), and 8517C $>$ T $($ AGC $>$ AGT); ORF8 mutation 251T $>$ C (TTA $>$ TCA $)$; N mutation 415T $>$ C 302 (TTG $>$ CTG); S mutation 1868A $>$ G $(\mathrm{GAT}>\mathrm{GGT})$; and ORF3a mutation $752 \mathrm{G}>\mathrm{T}(\mathrm{GGT}>\mathrm{GTT})$. Here, the 
304 ORF where the mutation is located.

Fig 7. Mutation profile for SARS-CoV-2 genomes (geolocation of China) with different HGS. Out of a total of 200 viral genomes, 36 genomes have unique HGS values. A total of 74 mutations were identified in all the genomes. The top 7 conserved mutations with were shown with special markers at the top of colored blocks representing ORFs. Mutation 10818G $>T$ in ORF1ab (codon TTG>TTT) occurred in populations with high HGS, which results in amino

311 acid M37F mutation in transmembrane protein nsp6. The mutation rarely occurred in 312 populations with low/moderate HGS.

314 Fig 8. Mutation profile for SARS-CoV-2 genomes (geolocation of the USA) with different

315 HGS. Out of a total of 1538 viral genomes, 140 genomes have unique HGS values. A total of $316 \mathbf{1 6 2}$ mutations were identified in all the genomes. The top 7 conserved mutations with were 317 shown with special markers at the top of colored blocks representing ORFs. Mutation 318 10818G $>$ T in ORF1ab (codon TTG>TTT) occurred in populations with high HGS, which 319 results in amino acid $\mathrm{M37F}$ mutation in transmembrane protein nsp6. The mutation rarely 320 occurred in populations with low/moderate HGS.

322 Fig 9. Mutation profile for SARS-CoV-2 genomes (geolocation of Europe) with different HGS. 323 Out of a total of 856 viral genomes, 98 genomes have unique HGS values. A total of 145 324 mutations were identified in all the genomes. The top 7 conserved mutations with were shown 
325 with special markers at the top of colored blocks representing ORFs. Mutation 10818G $>\mathrm{T}$ in

326 ORF1ab (codon TTG>TTT) occurred in populations with high HGS, which results in amino

327 acid M37F mutation in transmembrane protein nsp6. The mutation rarely occurred in

328 populations with low/moderate HGS.

329 Table 2. Conserved mutations identified in SARS-CoV-2 genomes with geolocations of China, the USA

330 and Europe.

331

\begin{tabular}{|c|c|c|c|c|c|c|}
\hline mutation & Location(ORF) & protein & $\begin{array}{l}\text { residue } \\
\text { mutation }\end{array}$ & residue type & residue property & Geolocation \\
\hline TTG $>$ TTT & 10818 & nsp6 & $\mathrm{M} 37 \mathrm{~F}$ & Phenylalanine & Hydrophobic & China \\
\hline GTA $>$ ATA & 1132 & nsp2 & V198I & Isoleucine & Hydrophobic & China \\
\hline $\mathrm{AGC}>\mathrm{AGT}$ & 8517 & nsp4 & S76S & Serine & Polar & China \\
\hline TTA $>$ TCA & 251 & ORF8 & L84S & Serine & Polar & China \\
\hline TTG $>$ CTG & 415 & $\mathrm{~N}$ & M139M & Methionine & Hydrophobic & China \\
\hline GAT $>$ GGT & 1868 & $\mathrm{~S}$ & D623G & Glycine & Special & China \\
\hline GGT $>$ GTT & 752 & ORF3a & G251V & Valine & Hydrophobic & China \\
\hline $\mathrm{ATC}>\mathrm{ACC}$ & 794 & nsp2 & $\mathrm{I} 85 \mathrm{~T}$ & Threonine & Polar & USA \\
\hline $\mathrm{CAT}>\mathrm{CAG}$ & 171 & ORF3a & H57Q & Glutamine & Polar & USA \\
\hline $\mathrm{TTT}>\mathrm{TTC}$ & 2772 & nsp3 & F106F & Phenylalanine & Hydrophobic & USA \\
\hline $\mathrm{CTT}>\mathrm{CCT}$ & 13859 & nsp12 & L228P & Proline & Special & USA \\
\hline GGT $>$ GAT & 1868 & $\mathrm{~S}$ & G623D & Aspartic acid & Negative & USA \\
\hline TTG $>$ TTT & 10818 & nsp6 & M37F & Phenylalanine & Hydrophobic & USA \\
\hline $\mathrm{AGC}>\mathrm{AGT}$ & 8517 & nsp4 & S76S & Serine & Polar & USA \\
\hline TTT $>$ TTC & 2772 & nsp3 & F106F & Phenylalanine & Hydrophobic & Europe \\
\hline $\mathrm{CTT}>\mathrm{CCT}$ & 13859 & nsp12 & L228P & Proline & Special & Europe \\
\hline TTG $>$ TTT & 10818 & nsp6 & M37F & Phenylalanine & Hydrophobic & Europe \\
\hline GGT $>$ GAT & 1868 & $\mathrm{~S}$ & G623D & Aspartic acid & Negative & Europe \\
\hline GGT $>$ GTT & 752 & ORF3a & G251V & Valine & Hydrophobic & Europe \\
\hline TAC $>$ TAT & 14256 & nsp12 & Y360Y & Tyrosine & Hydrophobic & Europe \\
\hline $\mathrm{CAG}>\mathrm{CAT}$ & 171 & ORF3a & Q57H & Histidine & Positive & Europe \\
\hline
\end{tabular}

332 Of all the gene mutations, the ORF1ab 10818G>T(TTG>TTT) mutation is the most interesting. This mutation

333 survived in all three regions (Fig 10). In addition, this mutation occurred only in the high HGS population

334 rather than in that with a lower HGS (Table 3). The SARS-CoV-2 ORF1ab gene encodes the precursor

335 polyprotein pp1ab, which is then cleaved into 16 nonstructural proteins (nsp1 to nsp16) by virus-encoded

336 proteinases. nsp6 plays a critical role in membrane anchoring of the RNA replication/transcription complex. 
337 The expression of the nonstructural protein nsp6 along with nsp3 and nsp4 mediates the formation of double-

338 membrane vesicles (DMVs)[33], which are organelle-like structures for viral genome replication and protect

339 against host cell defenses.

341 Fig 10. Highly conserved mutations identified in SARS-CoV-2 genomes with geolocations of

342 China, the USA and Europe. The three regions have different sets of mutations. The TTT (F,

343 Phenylalanine) mutation occurred in all three regions. TTT represents the mutation

344 10818G $>$ T(TTG>TTT) in ORF1ab. The $\mathbf{F}$ in the circle represents the amino acid mutation

345 M37F (Methionine to Phenylalanine) in nonstructural protein nsp6. The $\mathbf{P}, \mathbf{H},+,-$ and $\mathbf{S}$ in

346 brackets in the legend represent polar, hydrophobic, positively charged, negatively charged

347 and special residues, respectively.

349 Table 3. The mutation 10818G $>$ T in ORF1ab (codon TTG>TTT) of SARS-CoV-2 mostly occurs in

350 high-HGS (the first four columns) and rarely occurs in low-HGS population (the last four columns).

351 The top 15 genomes with high HGS are chosen as high-HGS population. The last 15 genomes with low

352 HGS are chosen as low-HGS population. GISAID accession ID and locations are given for genomes

353 from China, the USA and Europe. 


\begin{tabular}{|c|c|c|c|c|c|c|c|}
\hline Genome ID & HGS & Seq. (10813-10823) & Mut. & Genome ID & HGS & Seq. (10813-10823) & Mut. \\
\hline EPI-ISL-416331|China & 0.07085 & TTTTTTTATGA & $\mathrm{T}$ & EPI-ISL-406801|China & 0.06999 & TTTTTGTATGA & G \\
\hline EPI-ISL-431783|China & 0.07081 & TTTTTTTATGA & $\mathrm{T}$ & EPI-ISL-412982|China & 0.06986 & TTTTTGTATGA & G \\
\hline EPI-ISL-431180|China & 0.07080 & TTTTTGTATGA & G & EPI-ISL-421262|China & 0.06943 & TTTTTTTATGA & $\mathrm{T}$ \\
\hline EPI-ISL-416373|China & 0.07078 & TTTTTTTATGA & $\mathrm{T}$ & EPI-ISL-406534|China & 0.06942 & TTTTTGTATGA & G \\
\hline EPI-ISL-424360|China & 0.07074 & TTTTTGTATGA & G & EPI-ISL-413520|China & 0.06936 & TTTTTGTATGA & G \\
\hline EPI-ISL-405839|China & 0.07072 & TTTTTGTATGA & G & EPI-ISL-416397|China & 0.06935 & TTTTTGTATGA & G \\
\hline EPI-ISL-416325|China & 0.07071 & TTTTTGTATGA & G & EPI-ISL-421259|China & 0.06935 & TTTTTGTATGA & G \\
\hline EPI-ISL-402127|China & 0.07071 & TTTTTGTATGA & G & EPI-ISL-406533|China & 0.06934 & TTTTTGTATGA & G \\
\hline EPI-ISL-406595|China & 0.07069 & TTTTTGTATGA & G & EPI-ISL-421250|China & 0.06931 & TTTTTGTATGA & G \\
\hline EPI-ISL-408515|China & 0.07069 & TTTTTGTATGA & G & EPI-ISL-416330|China & 0.06930 & TTTTTGTATGA & G \\
\hline EPI-ISL-421253|China & 0.07069 & TTTTTGTATGA & G & EPI-ISL-418991|China & 0.06924 & TTTTTGTATGA & G \\
\hline EPI-ISL-412978|China & 0.07067 & TTTTTGTATGA & G & EPI-ISL-406798|China & 0.06922 & TTTTTGTATGA & G \\
\hline EPI-ISL-421256|China & 0.07066 & TTTTTGTATGA & G & EPI-ISL-416399|China & 0.06915 & TTTTTGTATGA & G \\
\hline EPI-ISL-412459|China & 0.07066 & TTTTTGTATGA & G & EPI-ISL-413749|China & 0.06914 & TTTTTGTATGA & G \\
\hline EPI-ISL-403932|China & 0.07065 & TTTTTGTATGA & G & EPI-ISL-421261|China & 0.06866 & TTTTTGTATGA & G \\
\hline EPI-ISL-427288|USA & 0.07205 & TTTTTTTATGA & $\mathrm{T}$ & EPI-ISL-437866|USA & 0.06931 & TTTTTGTATGA & G \\
\hline EPI-ISL-427190|USA & 0.07195 & TTTTTGTATGA & G & EPI-ISL-437384|USA & 0.06928 & TTTTTGTATGA & G \\
\hline EPI-ISL-435558|USA & 0.07153 & TTTTTTTATGA & $\mathrm{T}$ & EPI-ISL-424901|USA & 0.06928 & TTTTTGTATGA & G \\
\hline EPI-ISL-436064|USA & 0.07148 & TTTTTTTATGA & $\mathrm{T}$ & EPI-ISL-417353|USA & 0.06927 & TTTTTGTATGA & G \\
\hline EPI-ISL-430939|USA & 0.07144 & TTTTTTTATGA & $\mathrm{T}$ & EPI-ISL-445078|USA & 0.06923 & TTTTTGTATGA & G \\
\hline EPI-ISL-430404|USA & 0.07144 & TTTTTGTATGA & G & EPI-ISL-444068|USA & 0.06922 & TTTTTGTATGA & G \\
\hline EPI-ISL-406223|USA & 0.07142 & TTTTTTTATGA & $\mathrm{T}$ & EPI-ISL-429641|USA & 0.06921 & TTTTTGTATGA & G \\
\hline EPI-ISL-437857|USA & 0.07138 & TTTTTGTATGA & G & EPI-ISL-413458|USA & 0.06921 & TTTTTGTATGA & G \\
\hline EPI-ISL-444760|USA & 0.07138 & TTTTTGTATGA & G & EPI-ISL-427209|USA & 0.06915 & TTTTTGTATGA & G \\
\hline EPI-ISL-435444|USA & 0.07137 & TTTTTTTATGA & $\mathrm{T}$ & EPI-ISL-417453|USA & 0.06914 & TTTTTGTATGA & G \\
\hline EPI-ISL-428776|USA & 0.07133 & TTTTTGTATGA & G & EPI-ISL-416711|USA & 0.06914 & TTTTTGTATGA & G \\
\hline EPI-ISL-411956|USA & 0.07132 & TTTTTGTATGA & G & EPI-ISL-416677|USA & 0.06911 & TTTTTGTATGA & G \\
\hline EPI-ISL-418897|USA & 0.07128 & TTTTTGTATGA & G & EPI-ISL-429645|USA & 0.06910 & TTTTTGTATGA & G \\
\hline EPI-ISL-437799|USA & 0.07085 & TTTTTTTATGA & $\mathrm{T}$ & EPI-ISL-435562|USA & 0.06864 & TTTTTGTATGA & G \\
\hline EPI-ISL-422966|USA & 0.07081 & TTTTTTTATGA & $\mathrm{T}$ & EPI-ISL-427247|USA & 0.06856 & TTTTTGTATGA & G \\
\hline EPI-ISL-437322|Europe & 0.07226 & TTTTTTTATGA & $\mathrm{T}$ & EPI-ISL-445227|Europe & 0.06929 & TTTTTGTATGA & G \\
\hline EPI-ISL-437303|Europe & 0.07155 & TTTTTTTATGA & $\mathrm{T}$ & EPI-ISL-418264|Europe & 0.06925 & TTTTTGTATGA & G \\
\hline EPI-ISL-448774|Europe & 0.07151 & TTTTTGTATGA & G & EPI-ISL-447679|Europe & 0.06925 & TTTTTGTATGA & G \\
\hline EPI-ISL-447665|Europe & 0.07151 & TTTTTTTATGA & $\mathrm{T}$ & EPI-ISL-447510|Europe & 0.06925 & TTTTTGTATGA & G \\
\hline EPI-ISL-418243|Europe & 0.07149 & TTTTTTTATGA & $\mathrm{T}$ & EPI-ISL-413489|Europe & 0.06922 & TTTTTGTATGA & G \\
\hline EPI-ISL-430852|Europe & 0.07148 & TTTTTTTATGA & $\mathrm{T}$ & EPI-ISL-428688|Europe & 0.06922 & TTTTTGTATGA & G \\
\hline EPI-ISL-447654|Europe & 0.07148 & TTTTTTTATGA & $\mathrm{T}$ & EPI-ISL-428691|Europe & 0.06922 & TTTTTGTATGA & G \\
\hline EPI-ISL-420423|Europe & 0.07139 & TTTTTGTATGA & G & EPI-ISL-434619|Europe & 0.06921 & TTTTTGTATGA & G \\
\hline EPI-ISL-445241|Europe & 0.07139 & TTTTTGTATGA & G & EPI-ISL-445243|Europe & 0.06913 & TTTTTGTATGA & G \\
\hline EPI-ISL-434662|Europe & 0.07135 & TTTTTGTATGA & G & EPI-ISL-448468|Europe & 0.06910 & TTTTTGTATGA & G \\
\hline EPI-ISL-437896|Europe & 0.07133 & TTTTTGTATGA & G & EPI-ISL-434665|Europe & 0.06908 & TTTTTGTATGA & G \\
\hline EPI-ISL-447634|Europe & 0.07133 & TTTTTGTATGA & G & EPI-ISL-430859|Europe & 0.06867 & TTTTTGTATGA & G \\
\hline EPI-ISL-426379|Europe & 0.07081 & TTTTTTTATGA & $\mathrm{T}$ & EPI-ISL-418265|Europe & 0.06856 & TTTTTGTATGA & G \\
\hline EPI-ISL-448502|Europe & 0.07079 & TTTTTGTATGA & G & EPI-ISL-435144|Europe & 0.06848 & TTTTTGTATGA & G \\
\hline EPI-ISL-408430|Europe & 0.07078 & TTTTTTTATGA & $\mathrm{T}$ & EPI-ISL-437096|Europe & 0.06795 & TTTTTGTATGA & G \\
\hline
\end{tabular}


356 Studies on the nsp6 protein showed that the protein is a transmembrane protein with 6 transmembrane

357 regions[34]. This $10818 \mathrm{G}>\mathrm{T}$ ORF1ab mutation caused an amino acid mutation, M37F, in the nonstructural

358 protein nsp6, which is located in a loop between the first and second transmembrane domains on the N-

359 terminal side (Fig 11). This finding strongly suggested that the $10818 \mathrm{G}>\mathrm{T}$ (M37F) mutation survived a

360 selection event and resulted in a new population of SARS-CoV-2 with high HGS, which could be more

361 adapted to humans. In addition, the simultaneous occurrence of ORF1ab 10818G $>\mathrm{T}$ in all three regions

362 demonstrated that the mutation was highly stable in human-adapted strains. Although mutations in the

363 nonstructural proteins nsp4 and nsp6 may affect the assembly of DMVs and viral autophagy, the underlying

364 basis of how the M37F mutation results in SARS-CoV-2 adaptation in humans is not clear.

Fig 11. The topology of transmembrane protein nsp6 and the identified M37F mutation

side.

369 The identification of conserved mutations demonstrates that SARS-CoV-2 can improve host adaptation. It is

370 reasonable to hypothesize that high HGS in SARS-CoV-2 genomes and conserved mutations may explain

371 the epidemiological characteristics of COVID-19, such as mild symptoms, rapid spread and low mortality.

372 However, the mechanism behind the impairment remains poorly understood and calls for future laboratory

373 investigations. Viral genome data

\section{Discussion}

375 The HGS differences between SARS-CoV-2 and SARS-CoV genomes are critical to understanding clinical

376 manifestations of the ongoing pandemic. ORF $6,7 \mathrm{~b}, 8$ are the top 3 genes with significant HGS in SARS-

377 CoV-2. What's more, ORF 6 and ORF 8 of the SARS-CoV-2 have clear increments in HGS, up to about 
$378122 \%$ and $148 \%$ of that of SARS-CoV. Such apparent HGS changes suggest that these ORFs are important

379 in defining the difference between SARS-CoV-2 and SARS-CoV. In the ongoing SARS-CoV-2 pandemic,

380 the number of infected people is growing much faster than SARS and the total number of diagnosed cases

381 exceeded that of SARS. But the mortality rate (about $3 \%$ ) was lower than SARS (about 11\%)[35]. It is

382 known that the primary targets of SARS-CoV are lung and small intestine[36, 37]. Recent studies have found

383 that the SARS-CoV-2 may impair kidney function[38], infect the digestive system[39] and heart[40], and

384 cause liver damage[41]. Recent study showed that SARS-CoV-2 can cause thromboembolic

385 complications[42]. It has been reported that the SARS-CoV-2 virus can be found in stools and urine[40, 43].

386 In addition, an unusually long incubation period has been reported, during which more than half of the

387 patients had no signs of disease and the virus carriers may be highly contagious [43]. Why the COVID-19 is

388 so different from SARS is still not clear. But the mutations in virus genome and encoded proteins (such as

389 spike protein S) are believed as an important factor.

390 The knowledge on SARS-CoV-2 accessory proteins by now is quite limited. However, the viral genome and

391 proteins of SARS-CoV have been studied in depth in the past decade. Coronavirus has evolved to escape the

392 innate immune (especially IFN-I expression and signaling) through suppression of IFN induction and

393 singling pathways by non-structural proteins (nsps), structural proteins (S, E, M, N), and accessory proteins

394 (ORF 3a, 6, 7a, 7b, 8a, 8b) [20, 44-53]. By comparing the SARS-CoV gene HGS with that of SARS-CoV-2,

395 the obvious host-genome similarity changes shed light on the cause of rapid spread of COVID-19.

396 A power-law relationship is recognized between HGS and the expression of ISRE promoter, which is a direct

397 indicator of the virus to inhibit interferon synthesis. The HGS of ORF6 and ORF8 increase greatly in SARS-

$398 \mathrm{CoV}-2$, which represents enhanced ability in suppressing innate immune. Although the functions of accessory

399 proteins of SARS-CoV-2 have not been well studied, the secondary structure prediction reveals that ORF 6

400 and 8 are transmembrane proteins and may have related functions as in SARS-CoV. In fact, the SARS-CoV-2

401 contains a full-length ORF 8, which in SARS-CoV this reading frame is divided into ORF 8a and ORF 8b.

402 Linking of ORF 8a and ORF 8b into a single continuous gene fragment had no significant effect on virus 
404 to ORFs of SARS-CoV in function.

405 The discovery of increased HGS of ORF 6 and ORF 8 provide a strong evidence that SARS-COV-2 evolved

406 to be more adaptable to humans than SARS-CoV. Based on these findings, following conjecture is proposed

407 that the SARS-CoV-2 genes involved in suppressing the host's innate immunity are more powerful.

408 Therefore, SARS-CoV-2 causes the delayed response of host innate immunity, which results in rapid

409 transmission, low mortality and asymptomatic infection. These inferences are based on bioinformatics data,

410 but offer a valuable picture of how SARS-CoV-2 could become different from SARS-CoV. In addition, the

411 HGS method can also identify genetic mutations that help the virus adapt to humans.

412 It took the coronavirus 17 years to update from SARS-CoV to SARS-CoV-2. The significant increase in host-

413 genome similarity distinguishes SARS-COV-2 from SARS-COV. SARS-CoV-2 found out a way to improve

414 host adaptation. It is reasonable that high HGS may explain the quite different epidemiological characteristics

415 of SARS-CoV-2, such as mild symptoms, rapid spread and low mortality. But the mechanism behind the

416 impairment remains poorly understood and calls for future laboratory investigations. The COVID-19 appears

417 to be less able to cause deaths than SARS and MERS during the ongoing pandemic. However, there is still a

418 serious warning sign about viral mutation. The threat of another coronavirus outbreak with high

419 infectiousness and mortality remains an alarming possibility.

\section{$420 \quad$ Funding}

421 This work was supported by the C.C. Lin specific fund.

\section{Acknowledgments}

423 I thank the laboratories for sharing SARS-CoV-2 and SARS-CoV genomes through GISAID and

424 NCBI database. 


\section{References}

426 1.Wu F, Zhao S, Yu B, Chen YM, Wang W, Song ZG, et al. A new coronavirus associated with 427 human respiratory disease in China. Nature. 2020;579(7798):265-9. Epub 2020/02/06. PubMed 428 PMID: 32015508; PubMed Central PMCID: PMCPMC7094943.

429 2.Xu X, Chen P, Wang J, Feng J, Zhou H, Li X, et al. Evolution of the novel coronavirus from the 430 ongoing Wuhan outbreak and modeling of its spike protein for risk of human transmission. 431 SCIENCE CHINA Life Sciences. 2020.

432 3.He X, Lau EHY, Wu P, Deng X, Wang J, Hao X, et al. Temporal dynamics in viral shedding and 433 transmissibility of COVID-19. Nature Medicine. 2020;26(5):672-5.

434 4.Fiers W, Contreras R, Haegeman G, Rogiers R, Van de Voorde A, Van Heuverswyn H, et al. 435 Complete nucleotide sequence of SV40 DNA. Nature. 1978;273(5658):113-20.

436 5.Rosenberg M, Segal S, Kuff EL, Singer MF. The nucleotide sequence of repetitive monkey DNA 437 found in defective simian virus 40. Cell. 1977;11(4):845-57.

438 6.Senkevich TG, Bugert JJ, Sisler JR, Koonin EV, Darai G, Moss B. Genome sequence of a human 439 tumorigenic poxvirus: prediction of specific host response-evasion genes. Science (New York, 440 NY). 1996;273(5276):813-6. Epub 1996/08/09. PubMed PMID: 8670425.

441 7.Chang Y, Ma J, Zhang M, Yu Y. Prel iminary study on genome homology of viruses and human.

442 J N BETHUNE UNIV MED SCI. 1997;23(3):242-4.

443 8.Shackelton LA, Holmes EC. The evolution of large DNA viruses: combining genomic 444 information of viruses and their hosts. Trends in Microbiology. 2004;12(10):458-65.

445 9.Rouse BT, Sehrawat S. Immunity and immunopathology to viruses: what decides the outcome?

446 Nature Reviews Immunology. 2010;10(7):514-26. 
447 10.Van Kaer L, Joyce S. Viral evasion of antigen presentation: not just for peptides anymore.

$448 \quad$ Nature Immunology. 2006;7(8):795-7.

449 11.Enard D, Petrov DA. Evidence that RNA Viruses Drove Adaptive Introgression between

450 Neanderthals and Modern Humans. Cell. 2018;175(2):360-71.e13. PubMed PMID: 30290142.

451 12.Enard D, Cai L, Gwennap C, Petrov DA. Viruses are a dominant driver of protein adaptation in 452 mammals. Elife. 2016;5:e12469. PubMed PMID: 27187613.

453 13.Rothenburg S, Brennan G. Species-Specific Host-Virus Interactions: Implications for Viral

454 Host Range and Virulence. Trends in Microbiology. 2020;28(1):46-56.

455 14.NCBI RC. Database resources of the National Center for Biotechnology Information. Nucleic 456 acids research. 2018;46(D1):D8-d13. Epub 2017/11/16. PubMed PMID: 29140470; PubMed 457 Central PMCID: PMCPMC5753372.

458 15.Elbe S, Buckland-Merrett G. Data, disease and diplomacy: GISAID's innovative contribution to 459 global health. Global Challenges. 2017;1(1):33-46.

460 16.Benson DA, Cavanaugh M, Clark K, Karsch-Mizrachi I, Lipman DJ, Ostell J, et al. GenBank. 461 Nucleic acids research. 2013;41(Database issue):D36-42. Epub 2012/11/30. PubMed PMID: 462 23193287; PubMed Central PMCID: PMCPMC3531190.

463 17.Altschul SF, Gish W, Miller W, Myers EW, Lipman DJ. Basic local alignment search tool. 464 Journal of Molecular Biology. 1990;215(3):403-10.

465 18.Karlin S, Altschul SF. Methods for assessing the statistical significance of molecular sequence 466 features by using general scoring schemes. Proc Natl Acad Sci U S A. 1990;87(6):2264-8. PubMed 467 PMID: 2315319.

468 19.Frieman M, Yount B, Heise M, Kopecky-Bromberg SA, Palese P, Baric RS. Severe Acute 469 Respiratory Syndrome Coronavirus ORF6 Antagonizes STAT1 Function by Sequestering Nuclear 
470 Import Factors on the Rough Endoplasmic Reticulum/Golgi Membrane. Journal of Virology.

$471 \quad 2007 ; 81(18): 9812$.

472 20.Totura AL, Baric RS. SARS coronavirus pathogenesis: host innate immune responses and viral 473 antagonism of interferon. Current Opinion in Virology. 2012;2(3):264-75.

474 21.Pewe L, Zhou H, Netland J, Tangadu C, Olivares H, Shi L, et al. A SARS-CoV-specific protein 475 enhances virulence of an attenuated strain of mouse hepatitis virus. Advances in experimental 476 medicine and biology. 2006;581:493-8. Epub 2006/10/14. PubMed PMID: 17037583.

477 22.Chau TL, Gioia R, Gatot JS, Patrascu F, Carpentier I, Chapelle JP, et al. Are the IKKs and IKK478 related kinases TBK1 and IKK-epsilon similarly activated? Trends in biochemical sciences. 479 2008;33(4):171-80. Epub 2008/03/21. PubMed PMID: 18353649.

480 23.Guan Y, Zheng BJ, He YQ, Liu XL, Zhuang ZX, Cheung CL, et al. Isolation and 481 characterization of viruses related to the SARS coronavirus from animals in southern China. 482 Science (New York, NY). 2003;302(5643):276-8. Epub 2003/09/06. PubMed PMID: 12958366.

483 24.Keng C-T, Choi Y-W, Welkers MRA, Chan DZL, Shen S, Gee Lim S, et al. The human severe 484 acute respiratory syndrome coronavirus (SARS-CoV) $8 \mathrm{~b}$ protein is distinct from its counterpart in 485 animal SARS-CoV and down-regulates the expression of the envelope protein in infected cells. 486 Virology. 2006;354(1):132 - 42.

487 25.Wong HH, Fung TS, Fang S, Huang M, Le MT, Liu DX. Accessory proteins 8b and 8ab of 488 severe acute respiratory syndrome coronavirus suppress the interferon signaling pathway by 489 mediating ubiquitin-dependent rapid degradation of interferon regulatory factor 3. Virology. $490 \quad 2018 ; 515: 165-75$. 
491 26.Shi C-S, Nabar NR, Huang N-N, Kehrl JH. SARS-Coronavirus Open Reading Frame-8b

492 triggers intracellular stress pathways and activates NLRP3 inflammasomes. Cell Death Discov.

493 2019;5:101-. PubMed PMID: 31231549.

494 27.Lau SKP, Feng Y, Chen H, Luk HKH, Yang W-H, Li KSM, et al. Severe Acute Respiratory

495 Syndrome (SARS) Coronavirus ORF8 Protein Is Acquired from SARS-Related Coronavirus from

496 Greater Horseshoe Bats through Recombination. Journal of virology. 2015;89(20):10532-47. Epub

497 08/12. PubMed PMID: 26269185.

498 28.Le TM, Wong HH, Tay FPL, Fang S, Keng C-T, Tan YJ, et al. Expression, post-translational

499 modification and biochemical characterization of proteins encoded by subgenomic mRNA8 of the

500 severe acute respiratory syndrome coronavirus. The FEBS Journal. 2007;274(16):4211-22.

501 29.Kopecky-Bromberg SA, Martínez-Sobrido L, Frieman M, Baric RA, Palese P. Severe Acute

502 Respiratory Syndrome Coronavirus Open Reading Frame (ORF) 3b, ORF 6, and Nucleocapsid

503 Proteins Function as Interferon Antagonists. Journal of Virology. 2007;81(2):548.

504 30.Channappanavar R, Fehr Anthony R, Vijay R, Mack M, Zhao J, Meyerholz David K, et al.

505 Dysregulated Type I Interferon and Inflammatory Monocyte-Macrophage Responses Cause Lethal

506 Pneumonia in SARS-CoV-Infected Mice. Cell Host \& Microbe. 2016;19(2):181-93.

507 31.Wang C, Liu Z, Chen Z, Huang X, Xu M, He T, et al. The establishment of reference sequence

508 for SARS-CoV-2 and variation analysis. Journal of Medical Virology. 2020;92(6):667-74.

509 32.Pachetti M, Marini B, Benedetti F, Giudici F, Mauro E, Storici P, et al. Emerging SARS-CoV-

5102 mutation hot spots include a novel RNA-dependent-RNA polymerase variant. Journal of

511 translational medicine. 2020;18(1):179. Epub 2020/04/24. PubMed PMID: 32321524; PubMed

512 Central PMCID: PMCPMC7174922. 
513 33.Baliji S, Cammer SA, Sobral B, Baker SC. Detection of nonstructural protein 6 in murine

514 coronavirus-infected cells and analysis of the transmembrane topology by using bioinformatics and

515 molecular approaches. Journal of virology. 2009;83(13):6957-62. Epub 04/22. PubMed PMID:

51619386712.

517 34.Oostra M, Hagemeijer MC, van Gent M, Bekker CPJ, te Lintelo EG, Rottier PJM, et al.

518 Topology and Membrane Anchoring of the Coronavirus Replication Complex: Not All

519 Hydrophobic Domains of nsp3 and nsp6 Are Membrane Spanning. Journal of Virology.

$520 \quad 2008 ; 82(24): 12392$.

521 35.World Health Organization (2003) Consensus document on the epidemiology of severe acute

522 respiratory syndrome (SARS). Department of Communicable Disease Surveillance and Response,

523 WHO: 2003.

524 36.Lee N, Hui D, Wu A, Chan P, Cameron P, Joynt GM, et al. A major outbreak of severe acute 525 respiratory syndrome in Hong Kong. The New England journal of medicine. 2003;348(20):1986-

526 94. Epub 2003/04/16. doi: 10.1056/NEJMoa030685. PubMed PMID: 12682352.

527 37.Chan WS, Wu C, Chow SCS, Cheung T, To K-F, Leung W-K, et al. Coronaviral hypothetical

528 and structural proteins were found in the intestinal surface enterocytes and pneumocytes of severe

529 acute respiratory syndrome (SARS). Modern Pathology. 2005;18(11):1432-9. doi:

$530 \quad 10.1038 /$ modpathol.3800439.

531 38.Li Z, Wu M, Guo J, Yao J, Liao X, Song S, et al. Caution on Kidney Dysfunctions of 2019-

532 nCoV Patients. medRxiv. 2020:2020.02.08.20021212. doi: 10.1101/2020.02.08.20021212.

533 39.Zhang H, Kang Z, Gong H, Xu D, Wang J, Li Z, et al. The digestive system is a potential route 534 of 2019-nCov infection: a bioinformatics analysis based on single-cell transcriptomes. bioRxiv.

535 2020:2020.01.30.927806. doi: 10.1101/2020.01.30.927806. 
536 40.Zou X, Chen K, Zou J, Han P, Hao J, Han Z. The single-cell RNA-seq data analysis on the

537 receptor ACE2 expression reveals the potential risk of different human organs vulnerable to Wuhan 538 2019-nCoV infection Frontiers of Medicine 2020;online.

539 41.Chai X, Hu L, Zhang Y, Han W, Lu Z, Ke A, et al. Specific ACE2 Expression in Cholangiocytes

540 May Cause Liver Damage After 2019-nCoV Infection. bioRxiv. 2020:2020.02.03.931766. doi:

$541 \quad 10.1101 / 2020.02 .03 .931766$.

542 42.Lamamri M, Chebbi A, Mamane J, Abbad S, Munuzzolini M, Sarfati F, et al. Priapism in a 543 patient with coronavirus disease 2019 (COVID-19): A case report. Am J Emerg Med. 2020. doi:

544 10.1016/j.ajem.2020.06.027. PubMed PMID: PMC7301054.

545 43.Guan W-j, Ni Z-y, Hu Y, Liang W-h, Ou C-q, He J-x, et al. Clinical characteristics of 2019

546 novel coronavirus infection in China. medRxiv. 2020:2020.02.06.20020974. doi:

$547 \quad 10.1101 / 2020.02 .06 .20020974$.

548 44.Dosch SF, Mahajan SD, Collins AR. SARS coronavirus spike protein-induced innate immune 549 response occurs via activation of the NF- $\kappa$ B pathway in human monocyte macrophages in vitro.

550 Virus Research. 2009;142(1):19-27. doi: https://doi.org/10.1016/j.virusres.2009.01.005.

551 45.Fung TS, Liu DX. Human Coronavirus: Host-Pathogen Interaction. Annual review of 552 microbiology. 2019;73:529-57. Epub 2019/06/22. doi: 10.1146/annurev-micro-020518-115759.

553 PubMed PMID: 31226023.

554 46.Kikkert M. Innate Immune Evasion by Human Respiratory RNA Viruses. J Innate Immun. 555 2020;12(1):4-20. Epub 10/14. doi: 10.1159/000503030. PubMed PMID: 31610541.

556 47.Wong L-YR, Lui P-Y, Jin D-Y. A molecular arms race between host innate antiviral response 557 and emerging human coronaviruses. Virologica Sinica. 2016;31(1):12-23. doi: 10.1007/s12250$558 \quad 015-3683-3$ 
559 48.Zhang Q, Yoo D. Immune evasion of porcine enteric coronaviruses and viral modulation of

560 antiviral innate signaling. Virus Research. 2016;226:128-41. doi:

$561 \quad$ https://doi.org/10.1016/j.virusres.2016.05.015.

562 49.DeDiego ML, Nieto-Torres JL, Jimenez-Guardeño JM, Regla-Nava JA, Castaño-Rodriguez C,

563 Fernandez-Delgado R, et al. Coronavirus virulence genes with main focus on SARS-CoV envelope

564 gene. Virus Research. 2014;194:124-37. doi: https://doi.org/10.1016/j.virusres.2014.07.024.

565 50.Saitoh T, Akira S. Regulation of innate immune responses by autophagy-related proteins. J Cell

566 Biol. 2010;189(6):925-35. doi: 10.1083/jcb.201002021. PubMed PMID: 20548099.

567 51.Lim YX, Ng YL, Tam JP, Liu DX. Human Coronaviruses: A Review of Virus-Host Interactions.

568 Diseases. 2016;4(3):26. doi: 10.3390/diseases4030026. PubMed PMID: 28933406.

569 52.Nelemans T, Kikkert M. Viral Innate Immune Evasion and the Pathogenesis of Emerging RNA

570 Virus Infections. Viruses. 2019;11(10):961. doi: 10.3390/v11100961. PubMed PMID: 31635238.

571 53.MC F, GR S. Evasion of Host Innate Immunity by Emerging Viruses: Antagonizing Host RIG-I

572 Pathways. J Emerg Dis Virol. 2017;3(3):1-8.

573 54.Yount B, Roberts RS, Sims AC, Deming D, Frieman MB, Sparks J, et al. Severe Acute 574 Respiratory Syndrome Coronavirus Group-Specific Open Reading Frames Encode Nonessential 575 Functions for Replication in Cell Cultures and Mice. Journal of Virology. 2005;79(23):14909. doi:

$576 \quad$ 10.1128/JVI.79.23.14909-14922.2005. 
$580 \quad$ Figure captions

581 Fig 1. The HGS tree contains 200 SARS-CoV-2 genomes from China. Distance between leaves

582 is the unweighted pair distance between the 10-ORF-HGS vector of genomes. The color bar

583 represents the overall HGS value of each genome (weighted sum of ORF HGS). Out of a total

584 of 200 viral genomes, 36 have unique ORF HGS values. The histogram at the top left shows

585 the distribution of all genome HGS.

586 Fig 2. The HGS tree contains 1538 SARS-CoV-2 genomes from the USA. Distance between

587 leaves is the unweighted pair distance between the 10-ORF-HGS vector of genomes. The color

588 bar represents the overall HGS value of each genome (weighted sum of ORF HGS). Out of a

589 total of 1538 viral genomes, 140 have unique ORF HGS values. The histogram at the top left

590 shows the distribution of all genome HGS.

591 Fig 3. The HGS tree contains 856 SARS-CoV-2 genomes from Europe. Distance between

592 leaves is the unweighted pair distance between the 10-ORF-HGS vector of genomes. The color

593 bar represents the overall HGS value of each genome (weighted sum of ORF HGS). Out of a

594 total of 856 viral genomes, 98 have unique ORF HGS values. The histogram at the top left

595 shows the distribution of all genome HGS.

596 Fig 4. The HGS values of SARS-CoV-2 and SARS-CoV genes. ORF6 and ORF8 of SARS-

597 CoV-2 have apparently higher mean HGS values than those of SARS-CoV, reaching $122 \%$

598 and $148 \%$ of that of SARS-CoV ORF6 and ORF8, respectively.

599 Fig 5. SARS-CoV induced immune response in host cells. Host cell detect virus invasion 
600 mainly by TLPs and RIG1/MDA5 and lead to type I IFN signaling pathway. The receptor

601 IFNAR senses type I IFN and leads to the JAK1-STAT signaling pathway, which expresses

602 antiviral proteins and bring neighboring cell into anti-virus state. The ORF6 suppresses type

603 I IFN expression by inhibiting translocation of STAT1+STAT2+IRF9 complex into nucleus.

604 ORF 6 also circumvent IFN production by inhibit IRF-3 phosphorylation in signaling

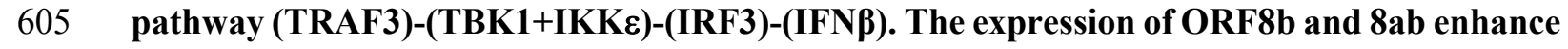

606 the IRF3 degradation, thus regulating immune functions of IRF3.

607 Fig 6. Inhibition of a promoter containing an ISRE by SARS-CoV proteins with different

608 genome HGS values. Cells were cotransfected with the SARS-CoV proteins and either

609 infected with Sendai virus (S. virus) or treated with IFN $\beta$ after 24 hours. The expression of

610 the promoter decays rapidly with the increasing HGS of ORF 3b, ORF 6 and N, conforming

611 to a power law.

612 Fig 7. Mutation profile for SARS-CoV-2 genomes (geolocation of China) with different HGS.

613 Out of a total of 200 viral genomes, 36 genomes have unique HGS values. A total of 74

614 mutations were identified in all the genomes. The top 7 conserved mutations with were shown

615 with special markers at the top of colored blocks representing ORFs. Mutation 10818G $>T$ in

616 ORF1ab (codon TTG>TTT) occurred in populations with high HGS, which results in amino

617 acid M37F mutation in transmembrane protein nsp6. The mutation rarely occurred in

618 populations with low/moderate HGS.

619 Fig 8. Mutation profile for SARS-CoV-2 genomes (geolocation of the USA) with different

620 HGS. Out of a total of 1538 viral genomes, 140 genomes have unique HGS values. A total of

$621 \quad \mathbf{1 6 2}$ mutations were identified in all the genomes. The top 7 conserved mutations with were 
622 shown with special markers at the top of colored blocks representing ORFs. Mutation

623 10818G $>$ T in ORF1ab (codon TTG $>$ TTT) occurred in populations with high HGS, which

624 results in amino acid $\mathbf{M 3 7 F}$ mutation in transmembrane protein nsp6. The mutation rarely

625 occurred in populations with low/moderate HGS.

626 Fig 9. Mutation profile for SARS-CoV-2 genomes (geolocation of Europe) with different HGS.

627 Out of a total of 856 viral genomes, 98 genomes have unique HGS values. A total of $\mathbf{1 4 5}$

628 mutations were identified in all the genomes. The top 7 conserved mutations with were shown

629 with special markers at the top of colored blocks representing ORFs. Mutation 10818G $>T$ in

630 ORF1ab (codon TTG>TTT) occurred in populations with high HGS, which results in amino

631 acid M37F mutation in transmembrane protein nsp6. The mutation rarely occurred in

632 populations with low/moderate HGS.

633 Fig 10. Highly conserved mutations identified in SARS-CoV-2 genomes with geolocations of

634 China, the USA and Europe. The three regions have different sets of mutations. The TTT (F,

635 Phenylalanine) mutation occurred in all three regions. TTT represents the mutation

636 10818G $>$ T(TTG>TTT) in ORF1ab. The $F$ in the circle represents the amino acid mutation

637 M37F (Methionine to Phenylalanine) in nonstructural protein nsp6. The $\mathbf{P}, \mathbf{H},+,-$ and $\mathbf{S}$ in

638 brackets in the legend represent polar, hydrophobic, positively charged, negatively charged

639 and special residues, respectively.

640 Fig 11. The topology of transmembrane protein nsp6 and the identified M37F mutation

641 located in a loop between the first and second transmembrane domains on the N-terminal

642 side. 
645 Dataset S1 (separate file). The accession number and corresponding HGS of 200 SARS-CoV-2 genomes with geolocation of China. Filename is DatasetS1_China_SARS-CoV2 nstrain200_ORFHGS_allinone.xls. The file contains accession ID $\overline{\mathrm{D}}$, collection date, location, 649 the weighted HGS of the whole genome.

650 Dataset S2 (separate file). The accession number and corresponding HGS of 1538 SARS-CoV-2 genomes with geolocation of the USA. Filename is DatasetS2_USA_SARS-CoV2_nstrain1538_ORFHGS_allinone.xls. The file contains accession ID, collection date, location, HGS values for 10 ORFs (ORF1ab, S, ORF3a, E, M, ORF6, ORF7a, ORF7b, ORF8, and N) and the weighted HGS of the whole genome.

Dataset S3 (separate file). The accession number and corresponding HGS of 856 SARS-CoV-2 genomes with geolocation of Europe. Filename is DatasetS3_Europe_SARS-CoV2_nstrain856_ORFHGS_allinone.xls. The file contains accession ID, collection date, location, HGS values for 10 ORFs (ORF1ab, S, ORF3a, E, M, ORF6, ORF7a, ORF7b, ORF8, and N) and the weighted HGS of the whole genome.

660 Dataset S4 (separate file). The accession number and corresponding HGS of 25 SARS-CoV 661 genomes. Filename is DatasetS4_SARS-CoV_nstrain25_ORFHGS_allinone.xls. The file 662 contains accession ID, HGS values for 10 ORFs (ORF1a $\bar{b}, \mathrm{~S}$, ORF3a, E, M, ORF6, ORF7a, 663 ORF7b, ORF8, and N) and the weighted HGS of the whole genome.

664 


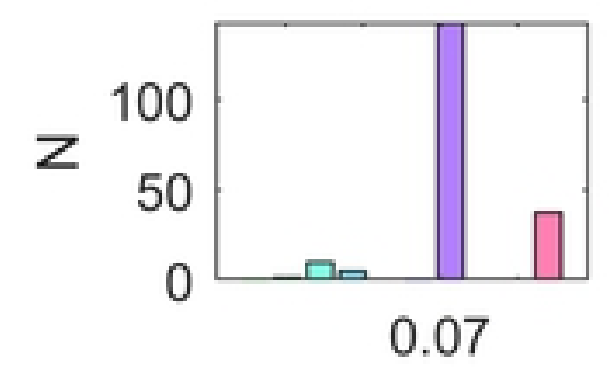

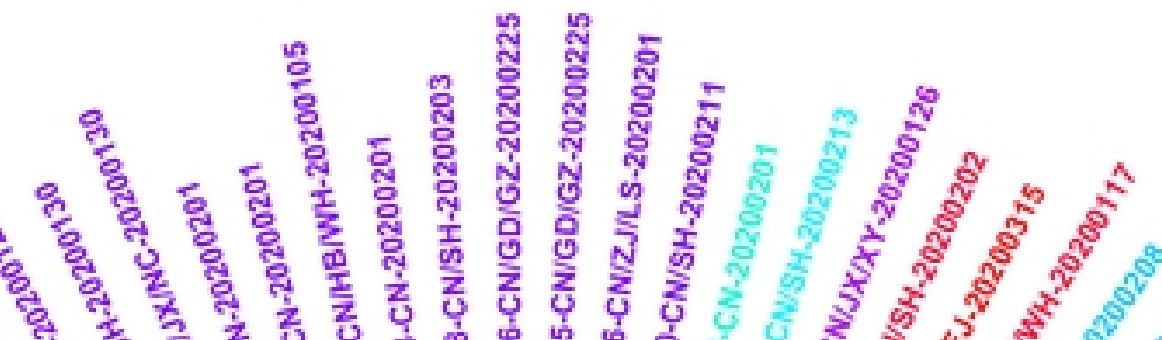

"8 \% 8 \%

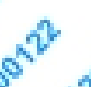

0.0706
0.0704
0.0702
0.07
0.0698

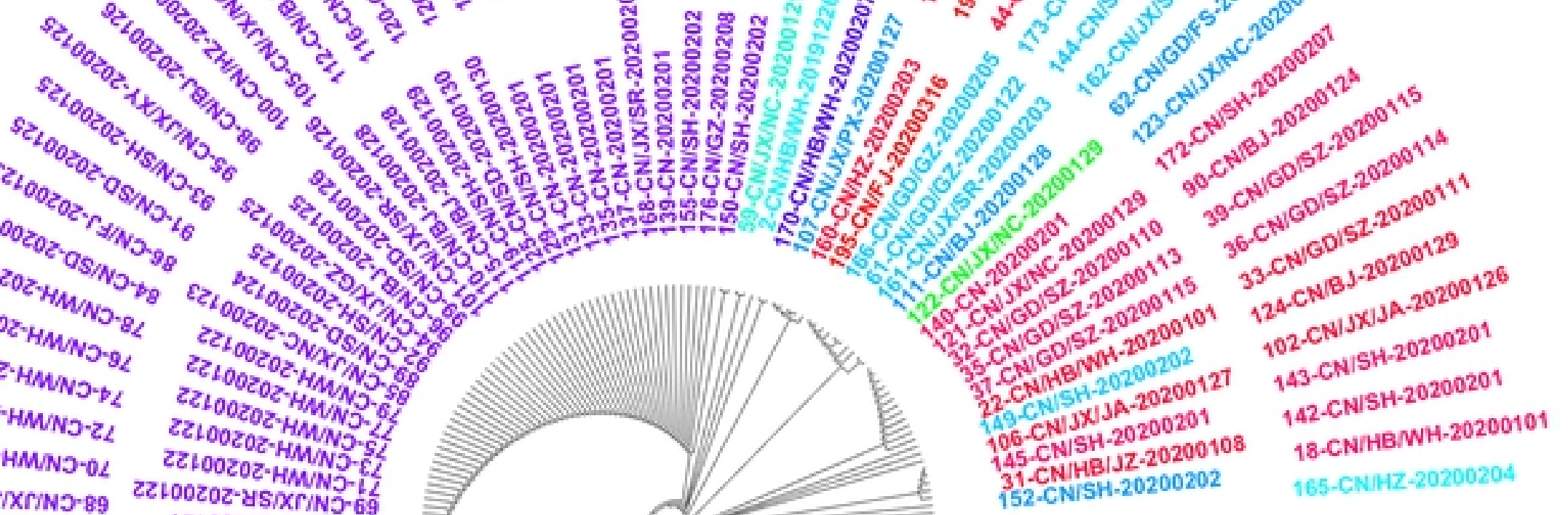

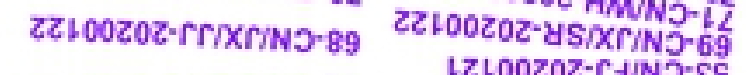

LILOOZ0Z-JNOJ/NO-Zs

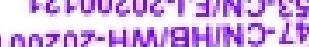

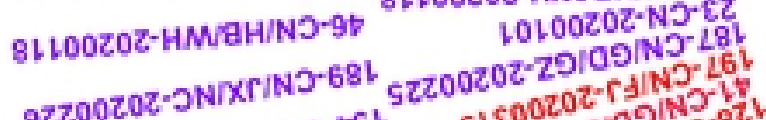

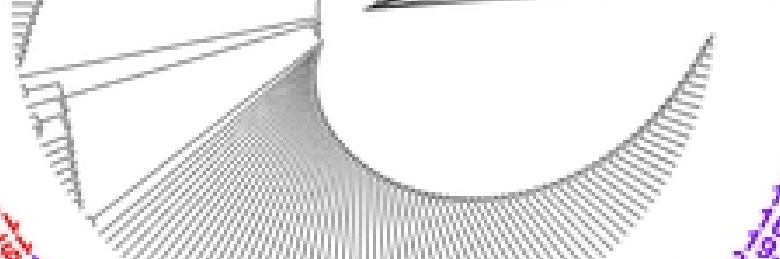

199.CN/NC-20200323 200-CN/GD/GZ-20200416 $10 . \mathrm{N}_{18} / \mathrm{WH}_{\mathrm{H}} 20191230$

4-CN/HB/WH.20191230 14.CN/W/WH-20191230

7. $\mathrm{C} N / \mathrm{HB} / \mathrm{WH} \cdot 20191230$

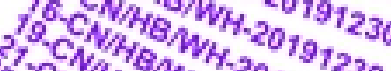

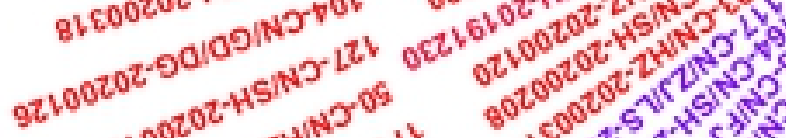

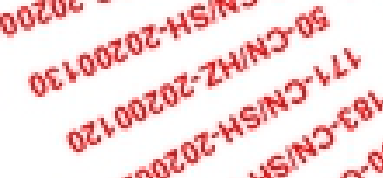

$\mathrm{N}=200$

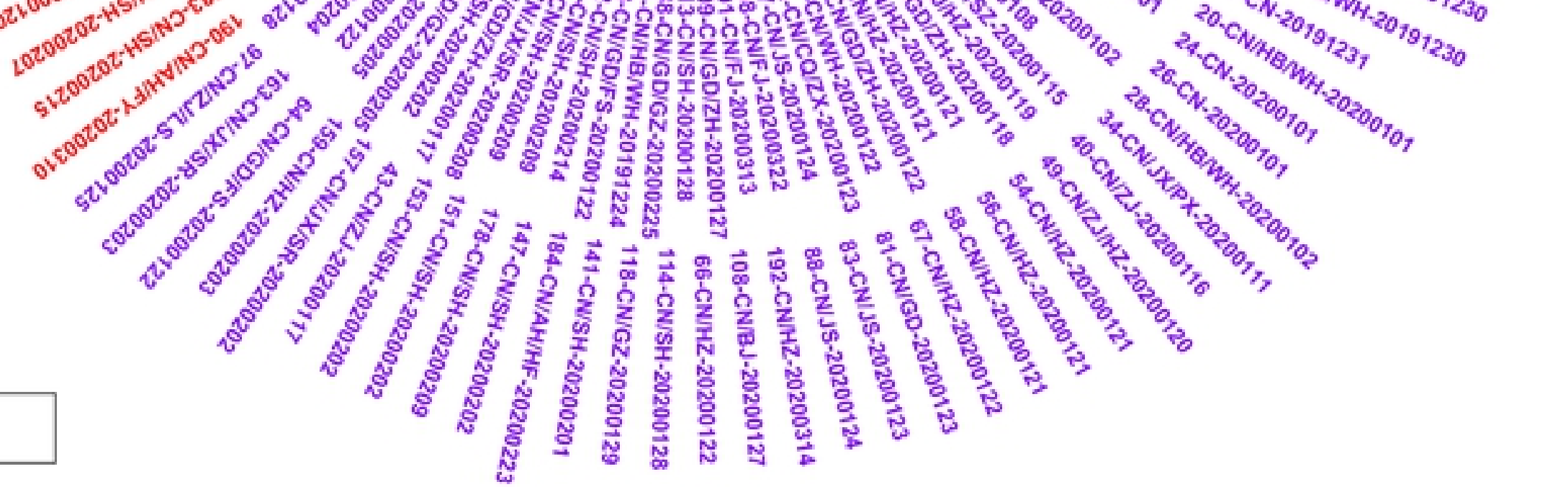

0.0692

0.069

0.0688

Fig1 


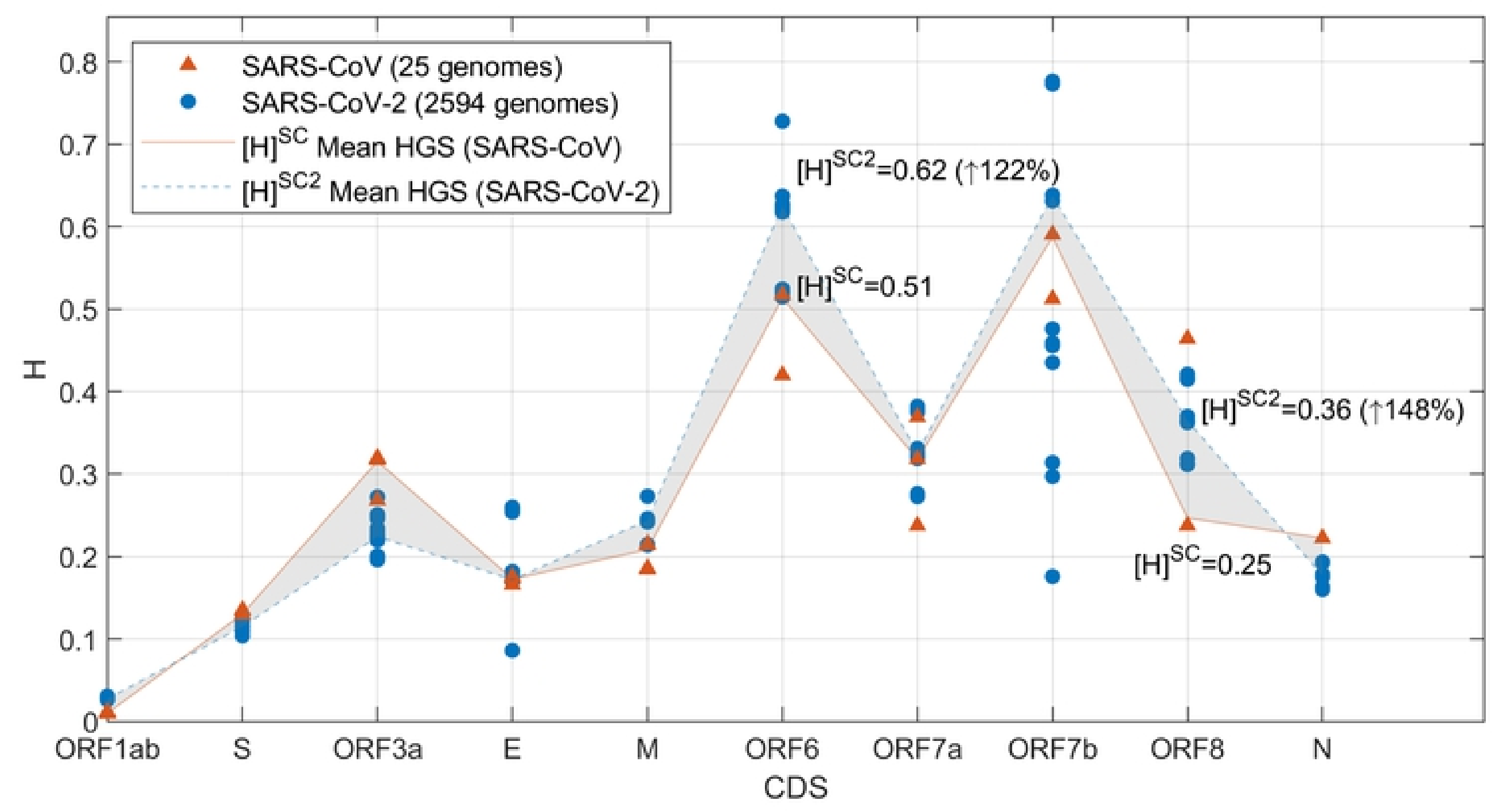

Fig4 

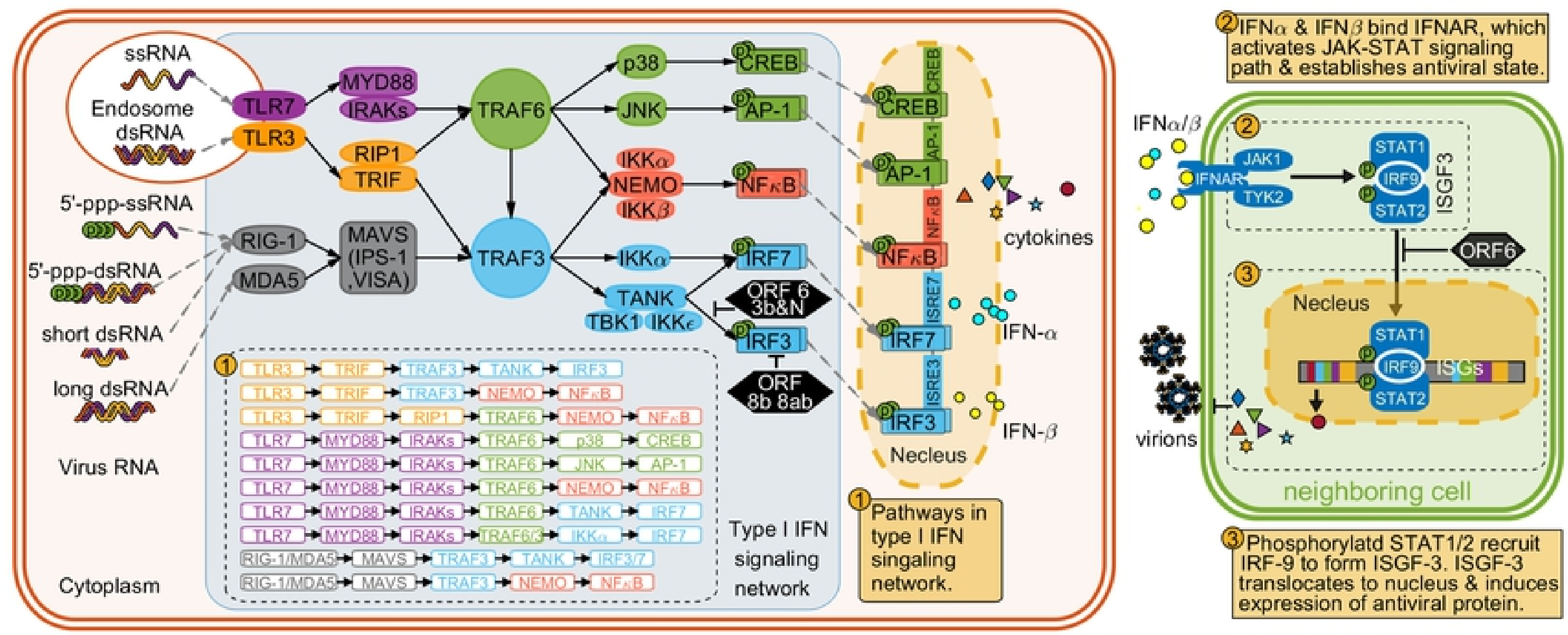

Fig5 


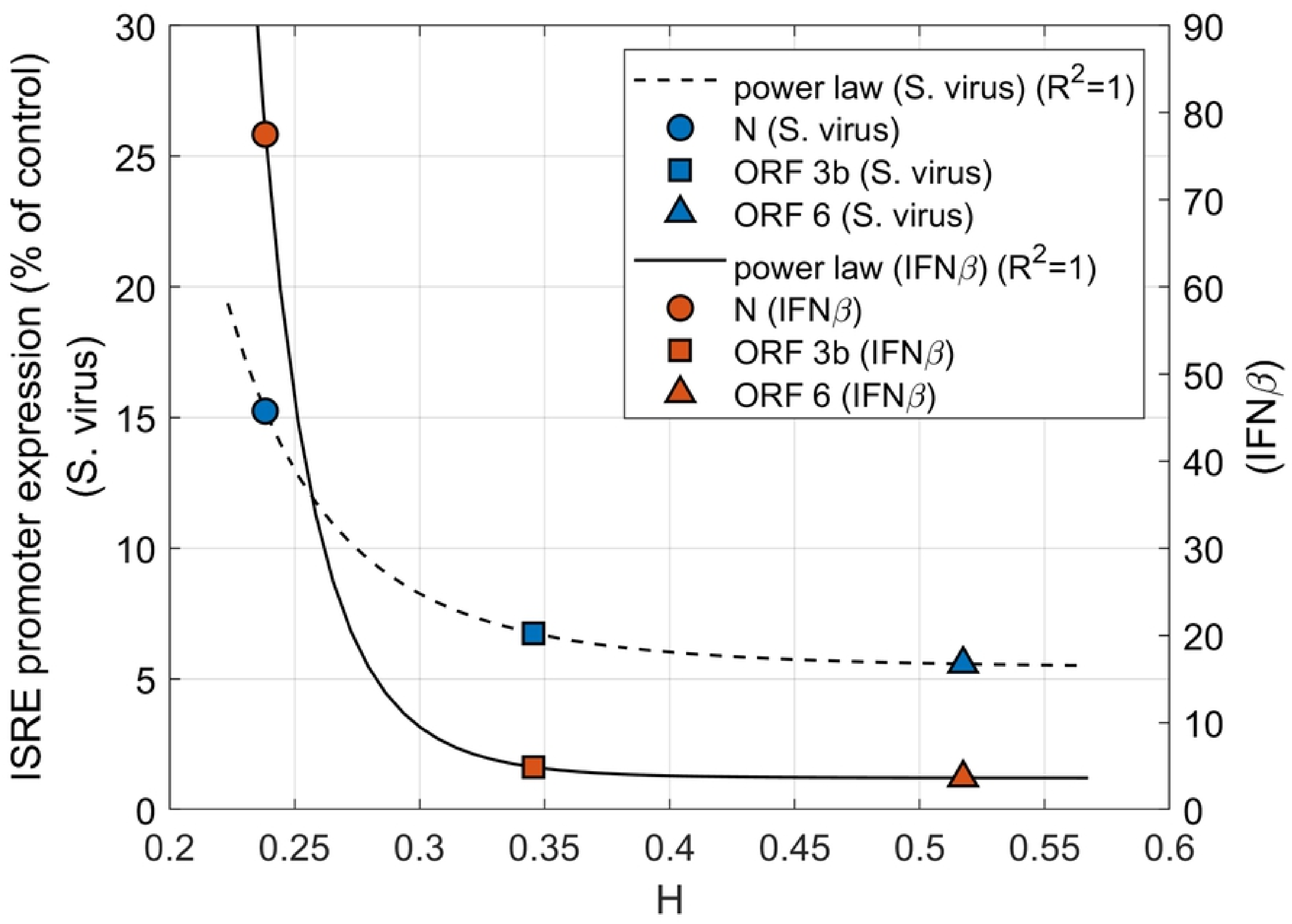

Fig6 


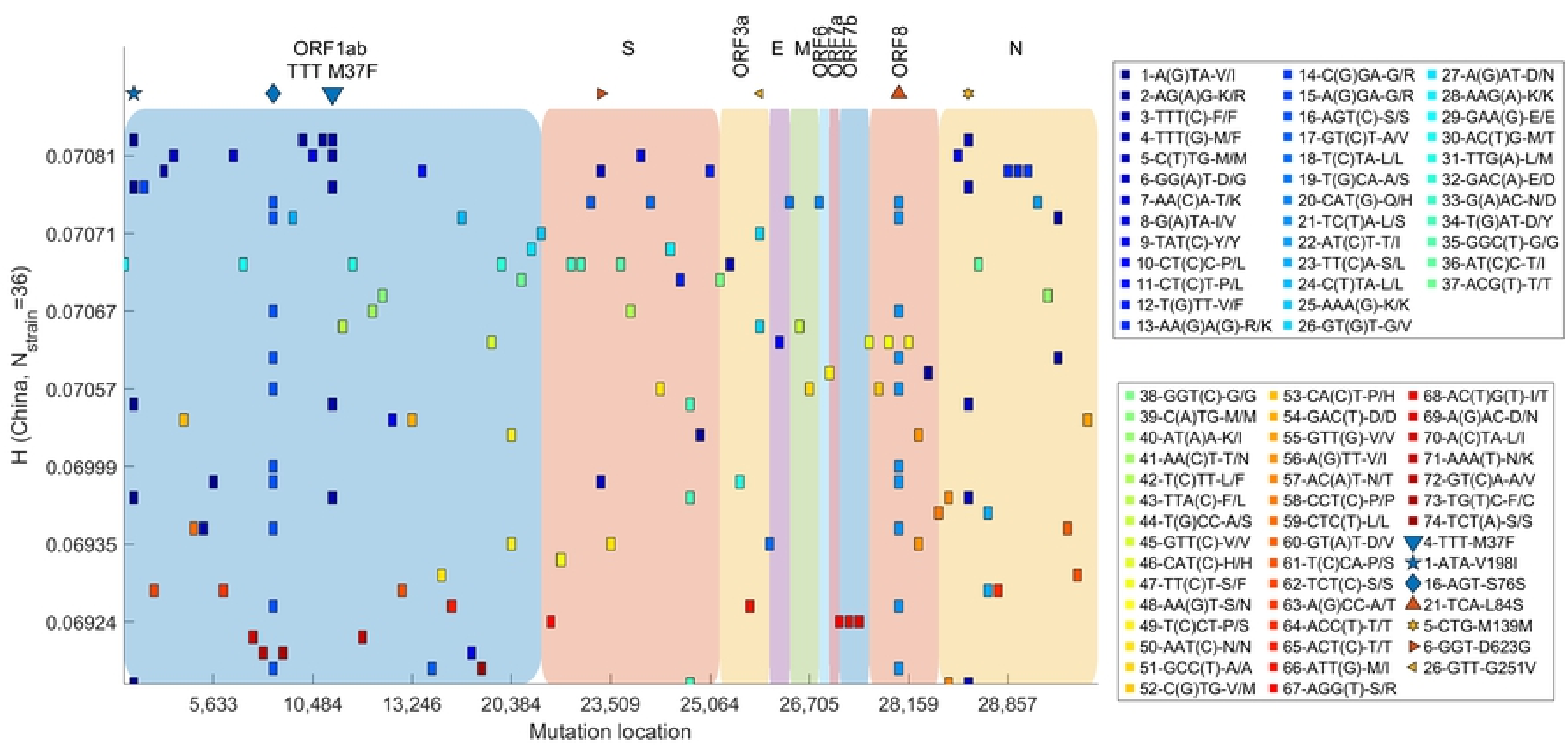

Fig7 


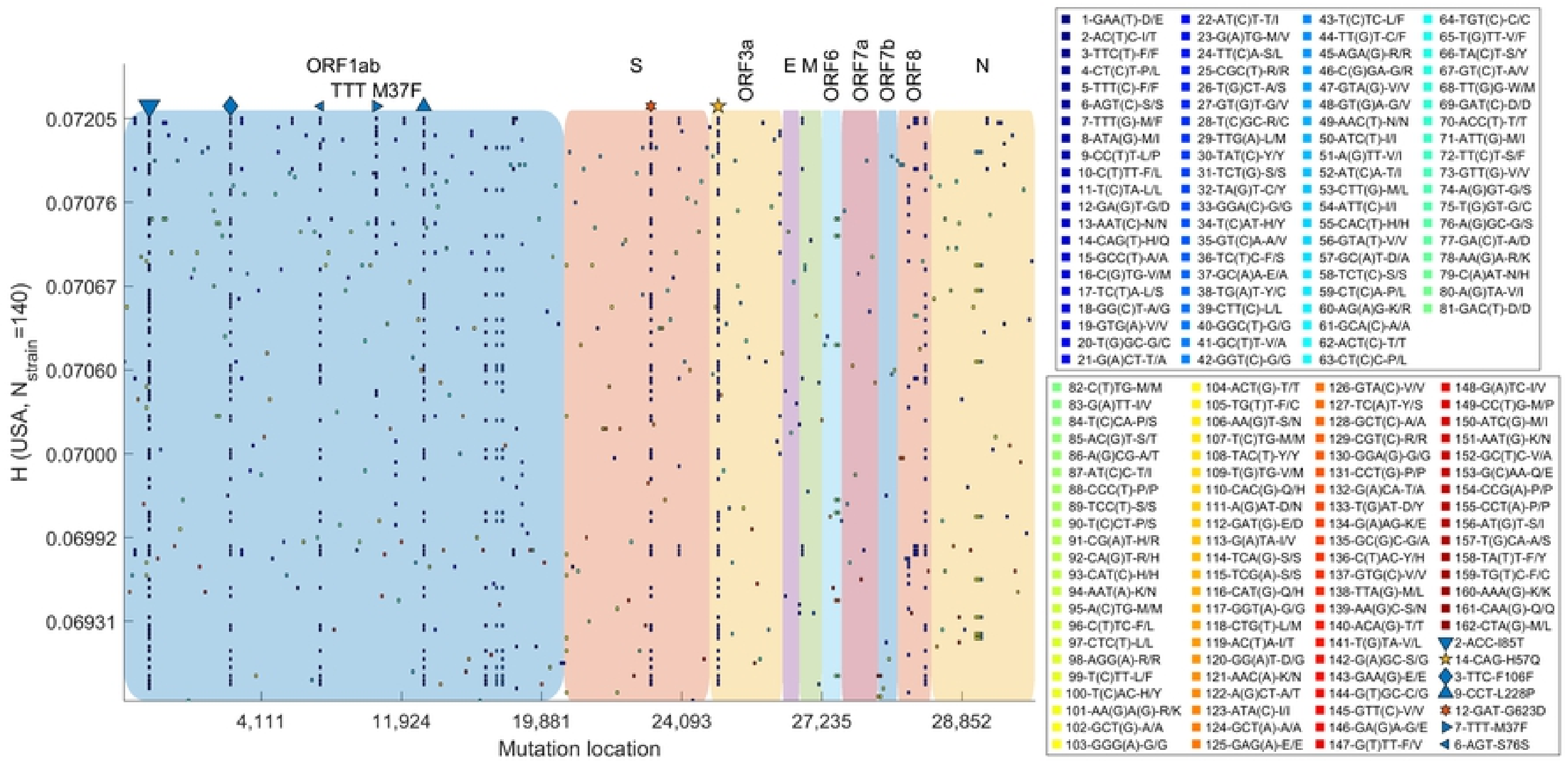

Fig8 


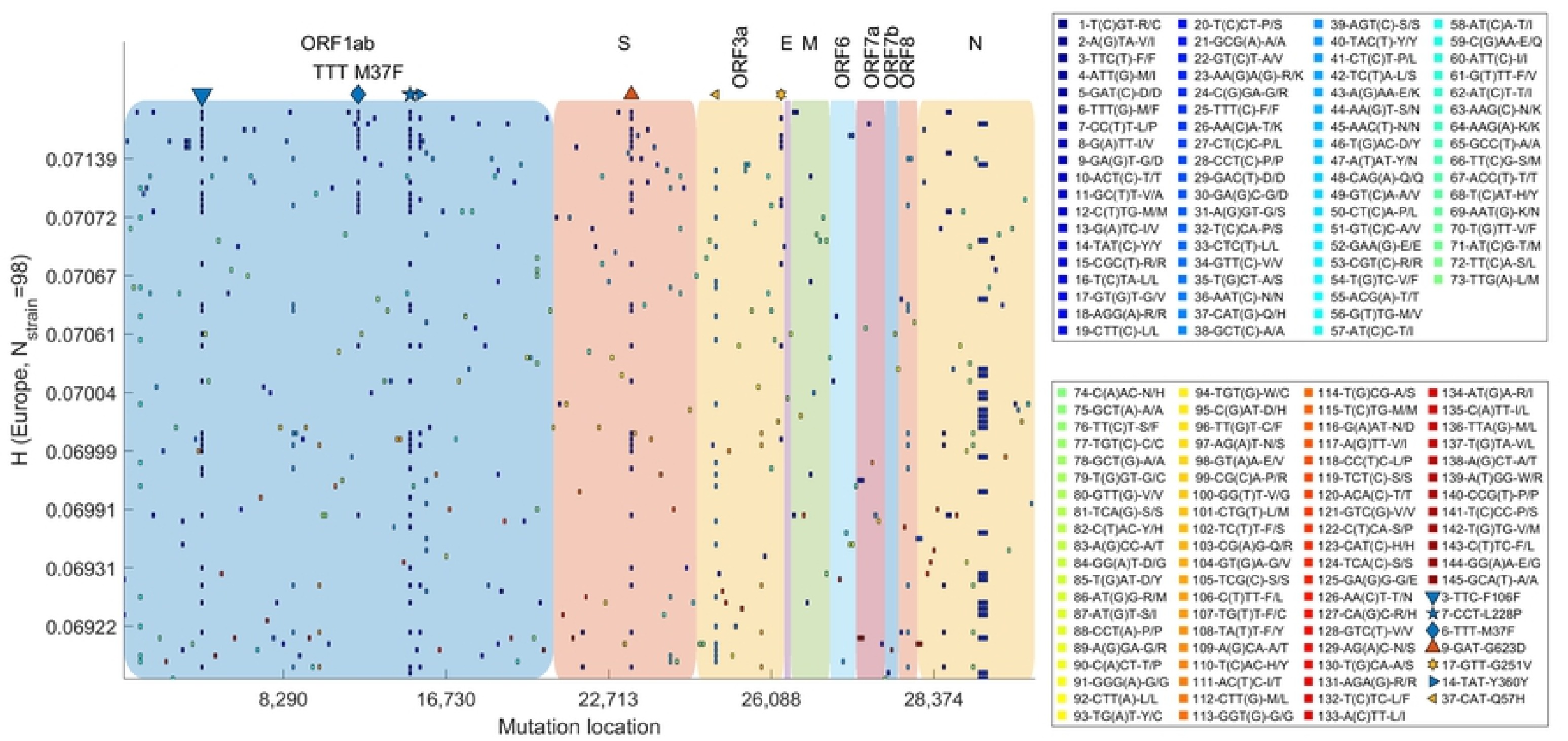

Fig9 


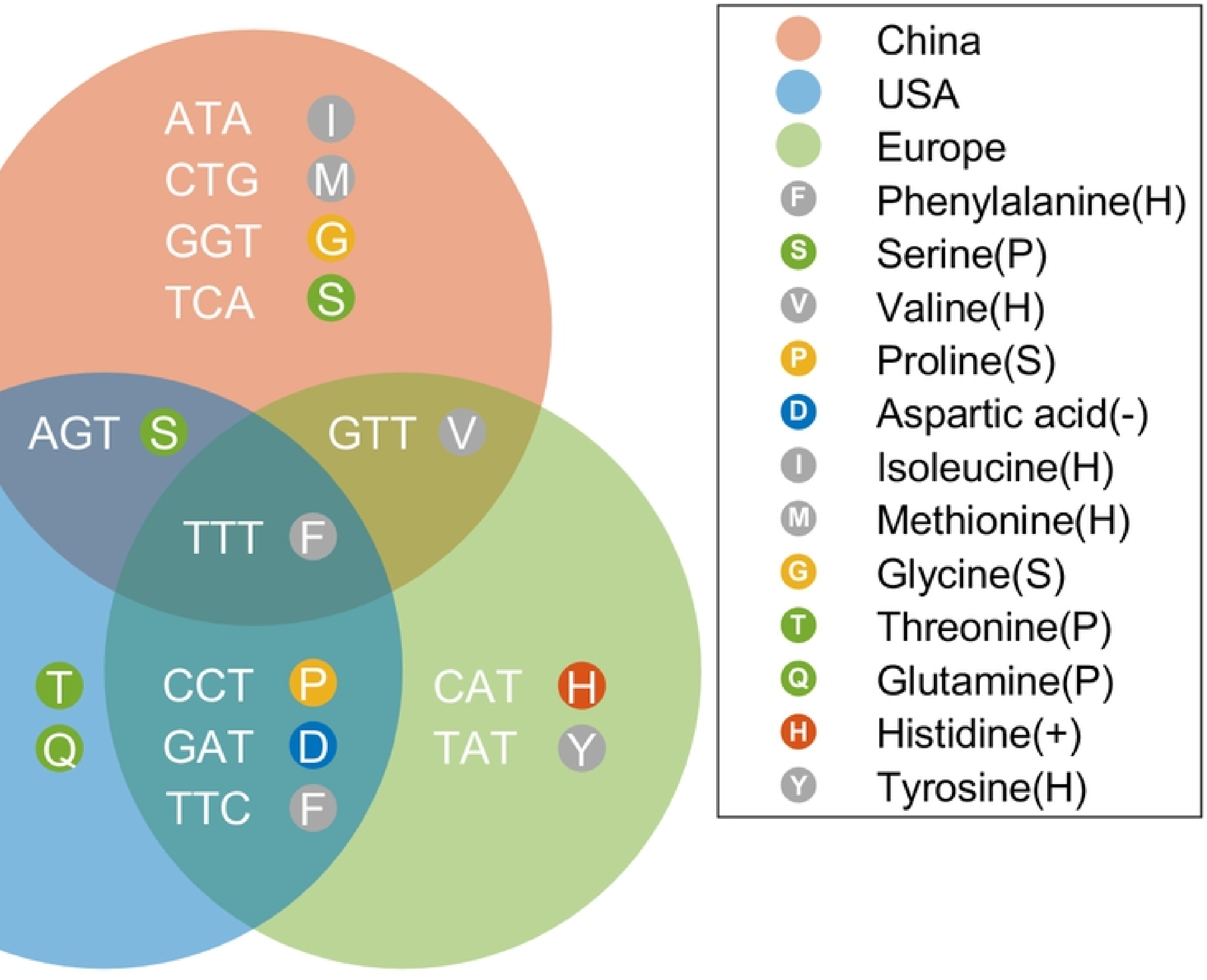

Fi10 


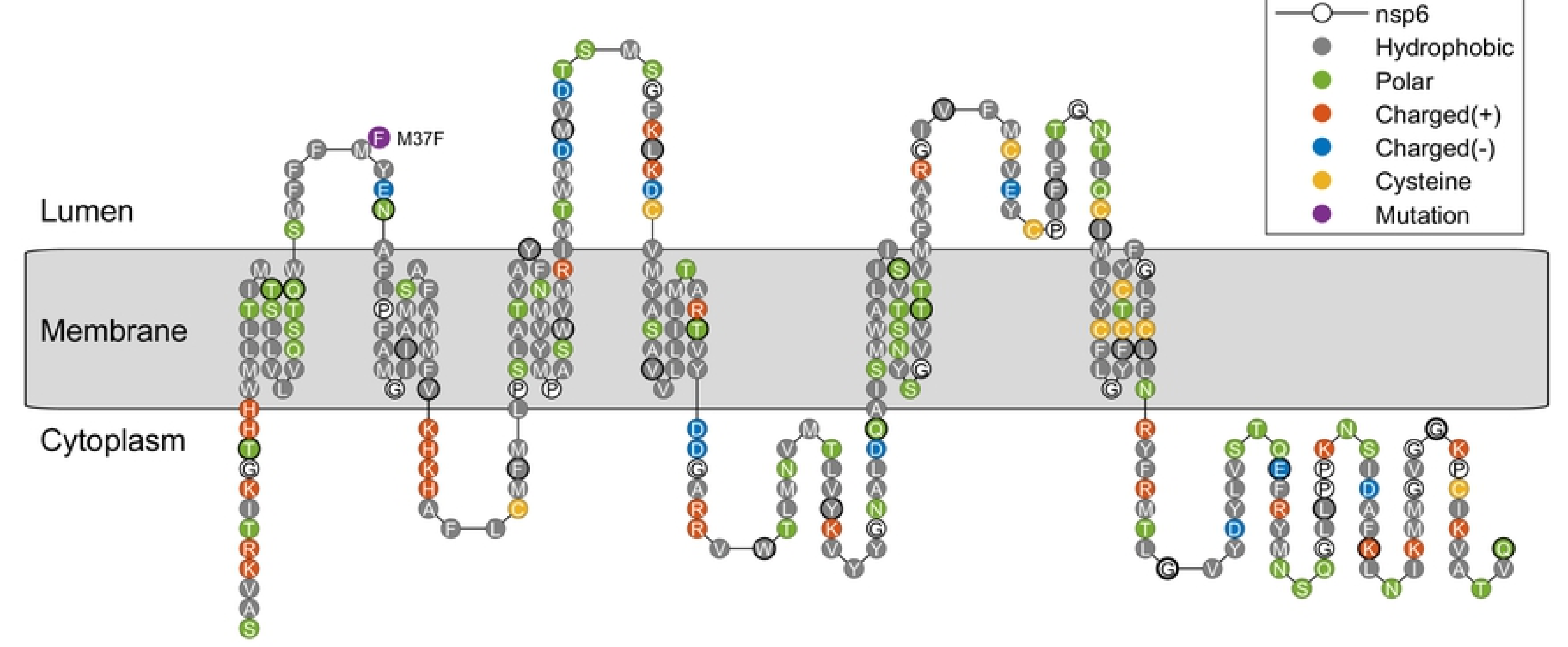

Fig11 